\title{
Parsimony Hierarchies For Inductive Inference *
}

\author{
Andris Ambainis \\ Institute of Mathematics and Computer Science \\ University of Latvia \\ Raina bulv. 29, Riga, LV-1459 \\ Latvia \\ John Case \\ Department of Computer and Information Sciences \\ University of Delaware \\ Newark, DE 19716 \\ USA \\ Mandayam Suraj \\ Department of Computer and Information Sciences \\ University of Delaware \\ Newark, DE 19716 \\ USA
}

\begin{abstract}
Freivalds defined an acceptable programming system independent criterion for learning programs for functions in which the final programs were required to be both correct and "nearly" minimal size, i.e, within a computable function of being purely minimal size. Kinber showed that this parsimony requirement on final programs limits learning power. However, in scientific inference, parsimony is considered highly desirable. A lim-computable function is (by definition) one calculable by a total procedure allowed to change its mind finitely many times about its output. Investigated is the possibility of assuaging somewhat the limitation on learning power resulting from requiring parsimonious final programs by use of criteria which require the final, correct programs to be "not-so-nearly" minimal size, e.g., to be within a lim-computable function of actual minimal size. It is shown that some parsimony in the final program is thereby retained, yet learning power strictly increases. Considered, then, are lim-computable functions as above but for which notations for constructive ordinals are used to bound the number of mind changes allowed regarding the output. This is a variant of an idea introduced by Freivalds and Smith. For this ordinal notation complexity bounded version of lim-computability, the power of the resultant learning criteria form finely graded, infinitely ramifying, infinite hierarchies intermediate between the computable and the lim-computable cases. Some of these hierarchies, for the natural notations determining them, are shown to be optimally tight.
\end{abstract}

Keywords: Computational Learning Theory, Minimal Size Program, Constructive Ordinal Notations, Limiting Computable Function.

AMS Subject Code Classification: 68Q32.

${ }^{*}$ The authors thank the anonymous referee for helpful comments, including simplification of some examples and proofs. Email addresses of the authors are: ambainis@lanet.lv, case@cis.udel.edu, sanjay@comp.nus.edu.sg, and suraj@cis.udel.edu, respectively. Grant support was received by A. Ambainis from Latvian Science Council Grant 01.0354, J. Case from NSF grant CCR-0208616, and by S. Jain from NUS grant R252-000-127-112. 


\section{Introduction}

This work is in the context of computability-theoretic inductive inference or learning theory [Put63, Gol67, JORS99, Odi99].

In Section 1.1 are informally presented what we need of the basic concepts and motivations from inductive inference including parsimony constraints on inferred (learned) programs.

As we will see, degrees of parsimony will be measured with notations from Kleene's $O$ for the constructive ordinals [Rog67]. Section 1.2 presents a corresponding informal introduction with motivations and examples.

Section 1.3 summarizes our principal results. These feature infinite hierarchies of success criteria re inferring parsimonious programs, and they are based on $O$-measured degrees of parsimony.

Section 2 contains the needed basic terminology and definitions presented rigorously.

Our main results are presented with proof in Section 3, and Section 4 presents further and preliminary results and open questions.

\subsection{Inductive Inference Machines and Parsimony}

$N$ denotes the set of natural numbers, $\{0,1,2,3, \ldots\}$. A basic paradigm from machine inductive inference pertains to the algorithmic, trial and error "learning" of programs for (computable) functions $f: N \rightarrow N$ - given, as input, enumerations of the successive values of $f$, i.e., $f(0), f(1), \ldots$. We can think of a function $f: N \rightarrow N$ as encoding the set of all possible deterministic outcomes of the experiments on some corresponding phenomenon (e.g., from chemistry): an input $x$ to $f$ represents the code of a possible experiment, e.g., mixing particular volumes of particular chemicals in a test tube, and $f(x)$ represents the outcome, e.g., the mixture turned blue and fizzed (see, for example, [BB75, CS83]). ${ }^{1}$ A program $p$ for such a function corresponds, then, to a predictive explanation for the associated phenomenon. Possession of $p$ enables one to predict the outcomes of any experiment (with associated code) $x$ regarding the phenomenon: use $p$ to compute $f(x)$ (and decode). The trial and error aspect of algorithmically learning such explanatory programs $p$ models that the scientific community changes its "mind" over time as to explanations for a phenomenon.

Informally, acceptable programming systems (synonym: acceptable numberings) [Rog58, Rog67, MY78, Roy87] are those programming systems for the partial computable functions which are intercompilable with naturally occurring general purpose programming formalisms such as Turing machine formalisms and the LISP programming language. ${ }^{2}$ The programs output by inductive inference algorithms (or machines) will be from some fixed acceptable system $\varphi \cdot \varphi_{p}$ denotes the partial computable function : $N \rightarrow N$ computed by the program $p$ in the $\varphi$-system. When an inductive inference machine $\mathbf{M}$ is given the successive values of a function $f$ as input and, after some resultant succession of (trial and error) output programs, it converges to a single program $p$, we write $\mathbf{M}(f)=p$. If this $p$ is a correct program (explanation) for $f$, i.e., if $\varphi_{p}=f$, we say that $\mathbf{M} \mathbf{E x}$-identifies $f$. If some $\mathbf{M} \mathbf{E x}$-identifies every function in a class of functions $\mathcal{C}$, we write $\mathcal{C} \in \mathbf{E x}$. For example, the class of primitive recursive functions is in $\mathbf{E x}$, and the class of computable functions is not [Gol67, BB75].

\footnotetext{
${ }^{1}$ Fulk [Ful85] argues that the set of possible, distinguishable experiments one can actually do on a phenomenon is countable.

${ }^{2}$ Typically, for theoretical work, one works with numerical names for the programs in these systems — whence the term 'numbering.'
} 
In scientific inference, parsimony of explanations is considered highly desirable. Program size is one of many ways to measure parsimony of programs [JORS99], and one can think of parsimony as a special case or variant of Occam's Razor. It is known, for computability-theoretic inductive inference, that requiring the final and correct programs to be minimal size [Sch98] is highly restrictive on inferring power [Fre75, Fre90] (and that the resultant inferring or learning power is dependent on which acceptable programming system is employed). Known too is the adverse effect on learning power of requiring the final and correct programs to be merely within a computable factor of minimal size [Kin74, Che82] (but that the resulting inferring power is independent of the underlying acceptable programming system [Fre75]). Hence, we see that, while parsimony is desirable, parsimony restrictions of even the weaker kind described just above limit inferring power. ${ }^{3}$

In the present study we will be exclusively concerned with criteria of success for inductive inference (parsimony restricted or not), and these criteria will be independent of the fixed underlying acceptable programming system from which the output hypotheses are drawn. For computable $f$, let $\operatorname{MinProg}(f) \stackrel{\text { def }}{=}$ the numerically least program for $f$. For $\mathcal{C} \in \mathbf{E x}$ as witnessed by $\mathbf{M}$, if there is a computable function $g$ such that, for every $f \in \mathcal{C}, \mathbf{M}(f) \leq g(\operatorname{MinProg}(f))$, then we say $\mathcal{C} \in \mathbf{M e x}$ (as witnessed by $\mathbf{M}$ and $g$ ). In this setting we call $g$ a parsimony factor. From [Kin74] we have, then, that Mex $\subset \mathbf{E x} .{ }^{4}$ For example, the set of functions which have value zero on almost all arguments $\in(\mathbf{E x}-\mathbf{M e x})$ [Kin74]. However, the class $\mathcal{S}_{0}$ of functions $f$ which, on input zero, output a program for $f$ is in Mex [Che82], and $\mathcal{S}_{0}$ is very large since, by the Kleene recursion theorem [Rog67], it contains a 1-variant of each computable function [BB75].

\subsection{Using Notations for Constructive Ordinals As Parsimony Measures}

To begin explaining the results of the present paper: a lim-computable function is one calculable by a total procedure allowed to change its mind finitely many times about its output. ${ }^{5}$

Let LimMex be the variant of Mex in which the parsimony factors $g$ can be lim-computable. It is noted in [Che82] that LimMex $\subset$ Ex. Hence, even the parsimony restriction for which the parsimony factors are allowed to be lim-computable lowers inferring or learning power compared with no parsimony restriction. However, we see, by Corollary 35 in Section 3 below, that Mex $\subset$ LimMex. Hence, while use of lim-computable parsimony factors lowers learning power compared with no parsimony restriction, use of lim-computable parsimony factors does not lower learning power as much as the use of computable parsimony factors. Therefore, the use of lim-computable parsimony factors partly assuages the limitation on learning power of parsimony restrictions compared with computable parsimony factors. With the use of lim-computable parsimony factors some desirable parsimony in the final programs is retained, yet learning power strictly increases over the use of computable parsimony factors.

As we described above, a (total) procedure that computes a lim-computable function is allowed to change its mind finitely many times about its output. One may, of course, consider the effects of restricting the number of mind changes of such procedures. In a different context, Putnam [Put65] studied mind change bounds $k \in N$ on the way to convergence of lim-computable characteristic functions and Ershov [Ers68a] showed these form a strict hierarchy in $k$.

\footnotetext{
${ }^{3}$ Case, Jain and Sharma [CJS96] study the effects on learning power of placing restrictions on the size differences between successive hypotheses output by an inductive inference machine. This topic is not pursued herein.

${ }^{4}$ ' $\subseteq$ ' denotes subset; ' $\subset$ ' denotes proper subset.

${ }^{5}$ Post [Sha71] first noticed that such functions characterize the functions computable from an oracle for the halting problem.
} 
Intuitively ordinals [Sie65, KM67] are representations of well-orderings. 0 represents the empty ordering, 1 represents the ordering of 0 by itself, 2 the ordering $0<1,3$ the ordering $0<1<2$, ... The ordinal $\omega$ represents the standard ordering of all of $N . \omega+1$ represents the ordering of $N$ consisting of the positive integers in standard order followed by $0 . \omega+\omega$ represents the ordering of $N$ consisting of the even numbers in standard order followed by the odd numbers in standard order. The constructive ordinals are just those that have a program (called a notation) in some system which specifies how to build them (lay them out end to end, so to speak). We will employ, as our system of notations, Kleene's general system $O$ [Kle38, Kle44, Kle55, Rog67, Sac90]. This system has at least one notation for each constructive ordinal and comes with Kleene's standard, useful order relation $<_{o}$ on the notations in $O .<_{o}$ naturally embeds into the ordering of the corresponding constructive ordinals.

Everyone knows how to use finite ordinals (the natural numbers) for counting, including for counting down. Freivalds and Smith [FS93] employed notations for constructive ordinals as devices for algorithmic counting down. ${ }^{6}$ They used such notations, for example, for algorithmic counting down the mind changes of inductive inference procedures. This allowed for handling and studying constructive, "transfinite" bounds on mind changes of inductive inference machines.

Herein we use notations $u$ from $O$ to bound the mind changes on the way to convergence of (total) procedures for lim-computable functions. The resultant functions we call $\lim _{u}$-computable. In Section 2.4 below we will formally define the associated $O$-countdown functions and use them to define formally the $\lim _{u}$-computable functions. Further below, in the current section, we present some informal examples. Of course we want to use $\lim _{u}$-computable functions as parsimony factors. Actually, for $u \in O$ for a positive constructive ordinal, to make the corresponding parsimony restricted inference criterion $\operatorname{Lim}_{u}$ Mex acceptable system independent, we need to employ monotonically non-decreasing $\lim _{u}$-computable parsimony factors. Of course it is natural anyhow to employ non-decreasing parsimony factors. Our main results present interesting infinite hierarchies for the criteria $\mathbf{L i m}_{u}$ Mex associated with particular transfinite subsequences of $u$ 's along certain natural $<_{o}$-paths. Thus, with the (non-decreasing) $\lim _{u}$-computable functions providing degrees of parsimony, we get infinite gradations in inferring or learning power.

In the present paper, we do not study the interesting connection between the $\lim _{u}$-computable functions and (transfinite) Ershov hierarchies [Add65, Ers68b, Ers70, EHK81, Sel84]. ${ }^{7}$ However, in [CS03], we show how to characterize the $\lim _{u}$-computable functions and variants in terms of concepts from [EHK81, Sel84]. ${ }^{8}$

The finite ordinals have unique notations in $O$ (see Section 2.3 below). For $n \in N$, we write $\underline{n}$ for the corresponding notation in $O$. The $\lim _{n}$-computable functions are just those for which an associated (total) mind changing procedure makes not more than $n$ mind changes.

Let $K$ be the diagonal halting problem set [Rog67]. Then, since $K$ is c.e. [Soa96] ${ }^{9}$, the charac-

\footnotetext{
${ }^{6}$ The results of [FS93] are also informally surveyed in [GS95].

Case, Jain and Suraj [CJS95] announced preliminary versions of some of the results of the present work, and herein are presented considerable improvements (and an emendation).

Subsequently to [CJS95], natural $O$-notations for constructive ordinals polynomial in $\omega$ have been extensively employed to provide natural classifications of standard problems within the context of learning grammars for formal languages [JS97, AJS99, JS99].

${ }^{7}$ The Ershov hierarchies are based on effective iteration of the Boolean operations including up into the constructive transfinite.

${ }^{8}$ While notations in $O$ as employed in [Ers68b, Ers70, EHK81, Sel84] are not directly treated as counters, in Jockusch's review [Joc82] of [EHK81] he provides the explicit intuition that a notation, as employed in [EHK81], serves as a kind of counter. This intuition is also explicit in [GD01].

${ }^{9}$ Where appropriate, we use "computably enumerable" (c.e.) and "computable" instead of "recursively enumer-
} 
teristic function of $K$ is $\lim _{\underline{1}}$-computable. ${ }^{10}$

The first limit ordinal, $\omega$, and each constructive ordinal larger than $\omega$, have infinitely many distinct notations in $O$. Suppose $w$ is any notation in $O$ for $\omega$. The $\lim _{w}$-computable functions are just those for which some mind changing procedure declares, if and when it makes its first mind change, the number of further mind changes that it may make about its output. Visually, think of $\omega$ as

$$
01223 \ldots n \ldots)
$$

where the right parenthesis can be thought of as an "open" right hand end marker. The corresponding counter starts at the right parenthesis (or at some number to the left of the right parenthesis); if and when there is a first mind change, the counter algorithmically leaps to some $n$ to the left; this leaves room for at most $n$ more mind changes with the counter moving to the left.

Let $F(x)=\max \left(\left\{\varphi_{e}(y) \mid \varphi_{e}(y)\right.\right.$ is defined $\left.\left.\wedge e, y \leq x\right\}\right)$. Clearly, for any notation $w$ in $O$ for $\omega, F(x)$ is $\lim _{w}$-computable, non-decreasing and not dominated by any computable function. By Lemma 22 below, $F$ is not $\lim _{\underline{\underline{n}}}$-computable.

For the next examples it is useful to discuss the very basic computable operations (for $O$ notations), $+_{o}, \times_{o}$, and $\{\cdot\}_{o}$. These operations on $O$ naturally embed into the corresponding (constructive) ordinal operations of addition, multiplication, and exponentiation, respectively. ${ }^{11}$ Just as Kleene essentially changed his definition of $+_{o}$ between [Kle38] and [Kle55] to obtain some auxiliary, useful properties, we have added an extra base case to his latter definition of $+_{o}$ to get useful properties we need. Our definition of $\times_{o}$ also features an extra base case over what would be needed merely to get the embedding into the corresponding ordinal operation. We did this too for technical usefulness. We guarantee thereby, for example, the technically helpful properties that, for all $y, \underline{0}+_{o} y=y, \underline{0} \times_{o} y=\underline{0}$, and $\underline{1} \times_{o} y=y$. In Section 2.5 below we present our "definitions" of $+_{o}, \times_{o}$, and $\{\cdot\}_{o}$ (as theorems) and present the resultant properties we need.

Convention 1 If $e$ is an expression evaluating to a number $n$ in $N$, then $\underline{e}$ is the unique notation for $n$ in the $O$ notation system.

Suppose $w \in O$ is for $\omega$ and $k \in N$. Of course $w+o \underline{k}$ is a notation for the ordinal $\omega+k$, which ordinal looks like a copy of the ordinal $\omega$ followed (on the right) by a copy of the ordinal $k$. The $\lim _{w+o \underline{k}}$-computable functions are those computed by (total) procedures which may make up to $k$ mind changes before they behave like a $\lim _{w}$-procedure. For $w+_{o} w=w \times_{o} \underline{2}$, the corresponding ordinal, $\omega+\omega=\omega \times 2$, looks like a copy of $\omega$ followed by a copy of $\omega$, i.e, like two copies of $\omega$ laid

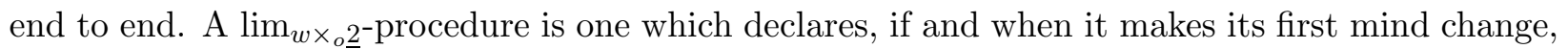
the number of further mind changes that it may make about its output, but can later revise this number once (possibly making a mind change with the revision too). We leave to the reader to work out the equivalence with visualizing a counter leaping and walking down from the right end - in a picture of $\omega \times 2$. For $n>2$, a $\lim _{w \times_{o} \underline{\underline{n}}}$-procedure is similar to a $\lim _{w \times_{o} \underline{2}}$-procedure, except that it can revise the number of further mind changes it may make $(n-1)$ times. $w \times_{o} w=\left\{w^{2}\right\}_{o}$, and the corresponding ordinal $\omega \times_{o} \omega=\omega^{2}$, can be visualized as $\omega$ copies of $\omega$ laid out end to end.

able" (r.e.) and "recursive", respectively, following recommendations in [Soa96].

${ }^{10}$ Recall that 1 is the notation in $O$ for the finite ordinal 1 .

${ }^{11}$ Intuitively, for $u, v \in O, u+_{o} v$ is a program for laying out the ordinal $|u|_{o}$ followed by the ordinal $|v|_{o}$ to form the ordinal $\left|u+_{o} v\right|_{o} . \times_{o}$, and $\left\{\cdot^{\circ}\right\}_{o}$ are for standardly iterating this action of $+_{o}$. 
A $\lim _{\{w-\}_{o}}$-procedure is one that can revise the number of further mind changes it may make " $\omega$ " times; that is, it can computably pick any number as the number of such revisions it can make. ${ }^{12}$

Suppose $w \in O$ is for $\omega$. For each $n>0$, we next give a fairly natural example below (based

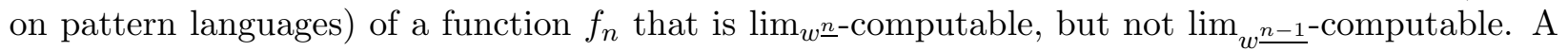
pattern language is (by definition) one generated by all the positive length substitution instances in a pattern, such as, for example, abXYcbbZXa - where the variables (for substitutions) are depicted in upper case and the constants/terminals in lower case. ${ }^{13}$ Let $D_{j}$ denote the finite set with canonical index $j$ and let $W_{i}$ be the c.e. set which is the domain of program $i$ in our fixed acceptable system [Rog67]. Suppose $n>0$. By careful modification of the proofs of [JS97, Theorem 1 and Corollary 1, pages 74-80], we have that, for each $n>0$, there exists a total function $f_{n}$ such that,

(a) for each $i, j$, if $W_{i}$ happens to be the union of 1 to $n$ pattern languages and $f_{n}(i)=j$, then $D_{j}$ is a set of (code numbers of) 1 to $n$ patterns, the union of whose corresponding pattern languages is $W_{i}$, and,

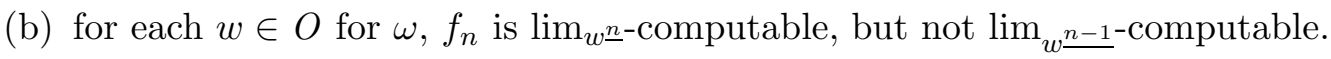

N.B. The notations featured above are natural ones for the corresponding ordinals - natural for those of us accustomed to thinking in terms of building ordinals from, for example, finite ordinals and $\omega$ by,$+ \times$, etc. From Theorem 55 below in Section 4.2 , we have, for example, a not so natural notation $v$ for $\omega^{2}$ such that $f_{3}$ from just above $i s \lim _{v}$-computable! This illustrates why we emphasize that our counters for counting down are notations for ordinals (and not ordinals themselves). ${ }^{14}$ On the other hand, as we note in the last paragraph of Section 4.2 below, by an obvious embedding, our Strong Hierarchy Theorem (Theorem 40 below) mutatis mutandis also holds for some weaker systems of notations than $O$, including non-maximal systems based on exponential polynomials in finite ordinals, $\omega, \ldots$. Such latter systems may have no "problematic" notations, but the corresponding hierarchies are not as extensive as those based on $O$. By Theorem 59 (also in Section 4.2) the interestingly "problematic" $O$-notations don’t appear for ordinals below $\omega^{2}$.

\subsection{Informal Summary of Results}

By Theorems 20 and 23 in Section 3, we have that, for all $n \in N, \mathbf{L i m}_{\underline{n}} \mathbf{M e x}=\mathbf{M e x}$ and, for all $u, u^{\prime} \in O$ and notations $w$ for $\omega$, such that $u \leq_{o} u^{\prime}<_{o} u \times_{o} w, \mathbf{L i m}_{u^{\prime}} \mathbf{M e x}=\mathbf{L i m}_{u} \mathbf{M e x}$. Hence, this suggests, for $\mathbf{L i m}_{u}$ Mex strict hierarchies, we should examine leaps of notation from $u$ to $u \times_{o} w$, where $w$ is a notation for $\omega$.

One of our main results, the Strong Hierarchy Theorem (Theorem 40 in Section 3 below) implies that the collapses of Theorems 20 and 23 are optimal for u's along certain natural $<_{o}-$ paths. In

\footnotetext{
${ }^{12}$ This characterization of counting down from $\left\{w^{\underline{2}}\right\}_{o}$ very much depends on the use of this particular notation for $\omega^{2}$. See below for more on such dependence on notation for ordinals $\omega^{2}$ and higher.

${ }^{13}$ The pattern languages were formally introduced by Angluin [Ang80]. Since then, much work has been done on pattern languages [Sal94a, Sal94b, $\mathrm{CJK}^{+} 01$ ] and finite unions thereof [Shi83, Wri89, KMU95, BUV96, CJLZ99]. Nix [Nix83] as well as Shinohara and Arikawa [SA95] outline interesting applications of pattern inference algorithms. For example, pattern language learning algorithms have been successfully applied toward some problems in molecular biology (see [SSS ${ }^{+}$94, SA95]). Pattern languages and finite unions of pattern languages turn out to be subclasses of Smullyan's [Smu61] Elementary Formal Systems (EFSs), and Arikawa, Shinohara and Yamamoto [ASY92] show that the EFSs can also be treated as a logic programming language over strings. The investigations of the learnability of subclasses of EFSs are interesting because they yield corresponding results about the learnability of subclasses of logic programs. Hence, these results have relevance for Inductive Logic Programming (ILP) [MR94, LD94, BM95, Mit97].

${ }^{14}$ Of course algorithmic counting down would seem to require working with a finite object/program such as a notation instead of a possibly infinite object such as an ordinal.
} 
particular it yields, for $v, w \in O$ where $w$ is for $\omega$,

(i) $\operatorname{Lim}_{\left\{w^{v}\right\}_{o}} \operatorname{Mex} \subset \operatorname{Lim}_{\left\{w^{v}\right\}_{o} \times_{o} w} \operatorname{Mex}$, and

(ii) $\left[v\right.$ is a notation for a limit ordinal $\left.\Rightarrow\left(\forall u<_{o} v\right)\left[\operatorname{Lim}_{\left\{w^{u}\right\}_{o}} \mathbf{M e x} \subset \mathbf{L i m}_{\left\{w^{v}\right\}_{o}} \mathbf{M e x}\right]\right]$.

Hence, for example, for $w \in O$ for $\omega$, Theorem 40 implies the hierarchy shown in Figure 1. ${ }^{15}$

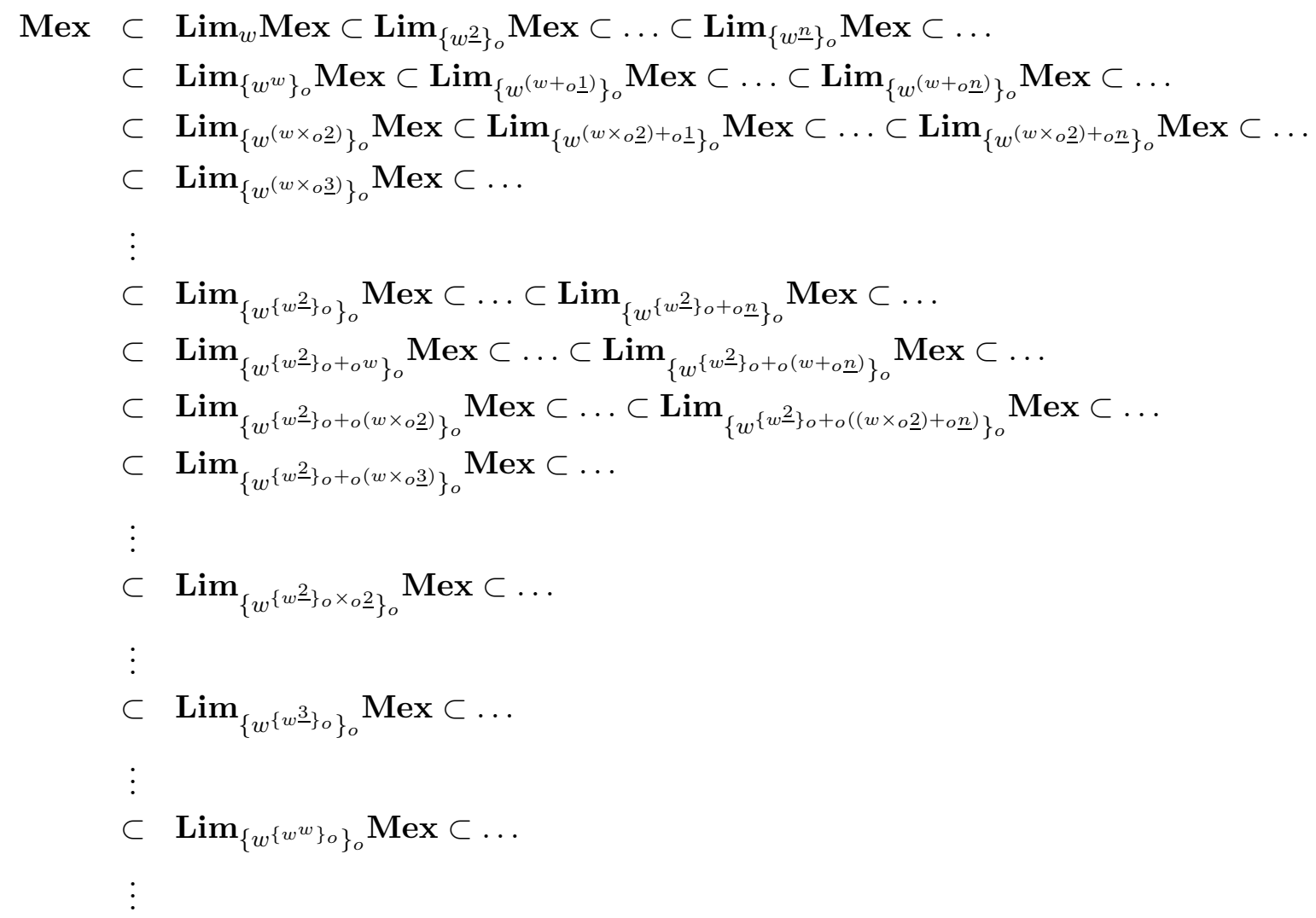

Figure 1: Example optimal hierarchy along a natural $<_{o}$-path for ordinals $<\epsilon_{0}$.

The hierarchy of Figure 1 extends, for example, to certain notations for the $\epsilon$ numbers and beyond too (see the discussion after Theorem 40 in Section 3 below).

Theorem 40 features notations in $O$ of the form $\left\{w^{v}\right\}_{o}$, where $w$ is for $\omega$. Our other main result, the General Hierarchy Theorem (Theorem 32 in Section 3 below), holds for any notations $u \in O$, but the hierarchy it specifies is not as tight as that of Theorem 40. Theorem 32 yields, for all $u \in O$, for all $w$ for $\omega, \operatorname{Lim}_{u} \operatorname{Mex} \subset \operatorname{Lim}_{\left\{(u+o \underline{2})^{w}\right\}_{o}}$ Mex.

Theorem 32 implies, for example, there are infinite, infinitely ramifying hierarchies of our parsimony restricted learning criteria which lie along $<_{o}$-paths, which paths have notations for each constructive ordinal (see the discussion following the proof of Theorem 32 and see Corollary 33). The use of $O$ (instead of other systems mentioned in this paper) enables us to have both Theorem 40 and Corollary 33.

\footnotetext{
${ }^{15}$ We note that by our carefully chosen definitions of $+_{o}, \times_{o}$, and $\{\cdot\}_{o}$, the following example equivalences, used in this hierarchy, hold: $w+_{o} w=w \times_{o} \underline{2}$ and $\left\{w^{w}\right\}_{o} \times_{o} w=\left\{w^{w+o \underline{1}}\right\}_{o}$.
} 
Section 4, which presents further and preliminary results and open questions, is divided into three sections.

Section 4.1 presents some preliminary results involving parsimony factors obtained by iterating the limit taking process. Theorems 47 and 49 essentially characterize all the criteria $\mathbf{L i m}_{u_{2}, u_{1}}^{2} \mathbf{M e x}$, where, for the associated parsimony factors, the first limit taken is restricted to $\leq_{o} u_{1}$ mind changes, and the second limit taken is restricted to $\leq_{o} u_{2}$ mind changes. For example, by Theorem 47, for notations $w_{2}, w_{1}$ for $\omega, \mathbf{L i m}_{w_{2}, w_{1}}^{2} \mathbf{M e x}=\mathbf{E x}$. Theorem 53 yields $\mathbf{L i m}_{\underline{1}, 2,2}^{3} \mathbf{M e x}=\mathbf{E x}$.

In Section 4.2, by Theorem 60, for $u, v \in O$ for the same ordinal $<\stackrel{\omega}{\omega}, \mathbf{L i m}_{u} \mathbf{M e x}=\mathbf{L i m}_{v} \mathbf{M e x}$, but, by Corollary 58, for each constructive ordinal $\alpha \geq \omega^{2}$, there exist notations $u, v$ for $\alpha$ such that $\mathbf{L i m}_{u} \operatorname{Mex} \neq \mathbf{L i m}_{v}$ Mex. It is also seen, in this same section, that if we define, for constructive ordinals $\alpha, \mathbf{L i m}_{\alpha} \operatorname{Mex} \stackrel{\text { def }}{=} \bigcup_{u}$ for $\alpha \operatorname{Lim}_{u} \mathbf{M e x}$, then there are only three distinct criteria of the form $\operatorname{Lim}_{\alpha} \mathbf{M e x}$, and they are in $\subset$-order: $\mathbf{M e x} \subset \operatorname{Lim}_{\omega} \mathbf{M e x} \subset \mathbf{L i m}_{\omega^{2}} \mathbf{M e x}$. This contrasts with the subtle and finely graded and ramified infinite $\mathbf{L i m}_{u} \mathbf{M e x}$-hierarchies based instead on $u$ 's $\in O$.

In Section 4.3 we recall the definition of the criteria $\mathbf{E x}_{u}$, for $u \in O$, essentially from [FS93]. This is a variant of $\mathbf{E x}$ where the learning or inductive inference machines themselves make $\leq_{o} u$ mind changes. From Theorems 62 and 66, we have that Mex $\nsubseteq \bigcup_{u \in O} \mathbf{E x}_{u}$, and, for all $u, w \in O$ such that $w$ is for $\omega, \mathbf{E x}_{w \times_{o}\left(u+_{o} \underline{1}\right)} \not \subset \mathbf{L i m}_{u} \mathbf{M e x}$. However, by Theorem 63, for all $u \in O$, for all $w$ for $\omega, \mathbf{E x}_{u} \subset \operatorname{Lim}_{\left\{(u+o \underline{2})^{w}\right\}_{o}} \mathbf{M e x}$. Also, by Theorem 64, for $u, v, w \in O$ such that $u<_{o}\left\{w^{v}\right\}_{o}$ and $w$ is for $\omega$, we have, $\mathbf{E x}_{u} \subset \mathbf{L i m}_{\left\{w^{v}\right\}_{o}} \mathbf{M e x}$.

\section{Preliminaries}

This section includes formal definitions from inductive inference, a description of notations for constructive ordinals, formal definitions for $O$-countdown functions and the $\mathbf{L i m}_{u} \mathbf{M e x}$ criteria mentioned above, and other necessary terminology.

\section{$2.1 \quad$ Notation}

As noted earlier, $N$ denotes the set of natural numbers, $\{0,1,2,3, \ldots\} . N^{+}$denotes the set of positive natural numbers, $\{1,2,3, \ldots\}$. $i, j, k, m, n, p, q, s, t, w, x, y, z$ (with or without subscripts, superscripts, ...) range over $N$. * denotes a non-member of $N$ such that $(\forall n \in N)[n<*<\infty](*$ represents 'unbounded but finite'). $u, v$, range over $N \cup\{*\} . X, Y, Z$, (with or without superscripts, subscripts, ...) range over subsets of $N$.

$\emptyset$ denotes the empty set. $\in, \notin, \subseteq, \subset, \supseteq$, つ respectively denote 'is a member of', 'is not a member of', 'is a subset of', 'is a proper subset of', 'is a superset of' and 'is a proper superset of'. $\uparrow$ denotes 'is undefined'. $\downarrow$ denotes 'is defined'.

For $S$, a subset of $N, \operatorname{card}(S)$ denotes the cardinality of $S$. So then, 'card $(S) \leq *$ ' means that $\operatorname{card}(S)$ is finite. $\max (S)$ and $\min (S)$ denote, respectively, the maximum and minimum of the set $S$, where $\max (\emptyset)=0$ and $\min (\emptyset)=\infty$. f,g,h and $F, G, H$ (with or without subscripts, superscripts, ...) range over total functions with arguments and values from $N$. We say that $g$ dominates $f \Leftrightarrow(\forall x)[g(x) \geq f(x)]$. $\psi$ ranges over partial functions with arguments and values from $N$. range $(\psi)$ denotes the range of $\psi$. The set of all total computable functions of one variable is denoted by $\mathcal{R}$. $\mathcal{C}, \mathcal{S}$ (with or without subscripts, superscripts, ...) range over subsets of $\mathcal{R}$. $P$ (with or without subscripts, superscripts, ...), ranges over predicates with arguments from $N$. 
$\langle\cdot, \cdot\rangle$ stands for an arbitrary, computable, one-to-one encoding of all pairs of natural numbers onto $N$ that is strictly monotonically increasing in both arguments [Rog67]. Similarly, $\langle\cdot, \ldots, \cdot\rangle$ can be used for encoding tuples of $n$ numbers onto $N . \pi_{i}^{n}$ denote the corresponding inverses: $\pi_{i}^{n}\left(\left\langle x_{1}, \ldots, x_{n}\right\rangle\right)=x_{i}$.

As in Section 1.1 above $\varphi$ denotes a fixed acceptable programming system. $W_{p}$ denotes the domain of $\varphi_{p} . \Phi$ denotes an arbitrary fixed Blum complexity measure for the $\varphi$-system [Blu67]. ${ }^{16}$

We let

$$
\varphi_{p, s}(x)= \begin{cases}\varphi_{p}(x), & \text { if } x \leq s \text { and } \Phi_{p}(x) \leq s \\ \uparrow, & \text { otherwise. }\end{cases}
$$

As defined earlier in Section 1.1, for a computable function $f, \operatorname{MinProg}(f) \stackrel{\text { def }}{=} \min \left(\left\{p \mid \varphi_{p}=f\right\}\right)$.

The quantifier ' $\forall{ }^{\infty}$ ' means 'for all but finitely many'; ' $\exists^{\infty}$ ' means 'there exists infinitely many'; and, ' $\exists$ !' means 'there exists a unique'.

\subsection{Inductive Inference Criteria and Identification}

In this section, we present relevant definitions and results from computability-theoretic learning theory.

If $\psi$ is defined at least on $0,1, \ldots, n-1$, then let $\psi[n]$ denote the sequence $\psi(0), \psi(1), \ldots, \psi(n-1)$. Let SEG be the set of sequences of natural numbers, i.e., the set of all $f[n]$, where $f \in \mathcal{R}$ and $n \in N$. A learning machine [Gol67, BB75, CS83] is a computable mapping from SEG into $N \cup\{$ ? .

Natural number outputs are interpreted as programs in the $\varphi$-system. Initially, a learning machine is allowed to output ?'s to indicate that it has not decided on its first program output yet, but once it outputs some program, it is not allowed to output ?'s again. We say that $\mathbf{M}(f)$ converges to $p$ (written $\mathbf{M}(f) \downarrow=p)$ iff $\left(\forall^{\infty} n\right)[\mathbf{M}(f[n])=p] ; \mathbf{M}(f)$ is undefined if no such $p$ exists (written $\mathbf{M}(f) \uparrow)$.

\section{Definition 2 (Gold [Gol67], Blum-Blum [BB75], Case-Smith [CS83])}

(a) $\mathbf{M} \mathbf{E x}$-identifies $f$ (written: $f \in \mathbf{E x}(\mathbf{M})) \stackrel{\text { def }}{\Leftrightarrow}\left(\exists i \mid \varphi_{i}=f\right)[\mathbf{M}(f) \downarrow=i]$.

(b) $\mathbf{E x}=\{\mathcal{S} \mid(\exists \mathbf{M})[\mathcal{S} \subseteq \mathbf{E x}(\mathbf{M})]\}$.

A machine $\mathbf{M}$ that $\mathbf{E x}$-identifies $f$ eventually converges to a program for $f$ in the $\varphi$ system. The criterion Ex is, however, independent of the acceptable programming system from which programs for functions are learned. ${ }^{17}$ In other words, if $\psi$ is any acceptable programming system, and $\mathbf{E x}_{\psi}$ is defined just as Ex is defined in Definition 2, except $\varphi$ is replaced by $\psi$ (therefore the outputs of inductive inference machines are interpreted as programs in the $\psi$ system), then $\mathbf{E x}_{\psi}=\mathbf{E x}$.

The criterion Mex (Definition 3 just below) is also independent of the underlying acceptable programming system [Fre75].

Definition 3 (Freivalds [Fre75], Chen [Che82])

(a) Suppose $g$ is a total (not necessarily computable) monotonically non-decreasing function. Then, a learning machine $\mathbf{M} g$-Mex-identifies $f$ (written: $f \in g$ - $\mathbf{M e x}(\mathbf{M})) \stackrel{\text { def }}{\Leftrightarrow} \mathbf{M} \mathbf{E x}$-identifies $f$ and $\mathbf{M}(f) \leq g(\operatorname{MinProg}(f))$.

\footnotetext{
${ }^{16}$ For example, if $\varphi$ is based on Turing machines, we could take $\Phi_{p}(x)$ to be the number of Turing machine steps Turing machine $p$ executes on input $x$ (undefined if infinite).

${ }^{17}$ This result easily follows from the definition of Ex and the fact that any two acceptable programming systems are computably isomorphic [Rog58, Rog67].
} 
(b) A learning machine $\mathbf{M}$ Mex-identifies $\mathcal{S} \stackrel{\text { def }}{\Leftrightarrow}$ there exists a computable monotonically nondecreasing $g$ such that $\mathcal{S} \subseteq g-\mathbf{M e x}(\mathbf{M})$.

(c) $\mathbf{M e x}=\{\mathcal{S} \mid(\exists \mathbf{M})[\mathbf{M}$ Mex-identifies $\mathcal{S}]\}$.

In the definition just above, the $g$ 's represent parsimony factors by which the parsimony constraints are loosened. The final programs are, in a sense, nearly-minimal-size. Kinber was first [Kin74, Fre75] to show that Mex $\subset$ Ex.

Nearly minimal size program inference, as defined by Mex, requires that the final program size be within a computable parsimony factor of the actual minimum program. In the present paper, we relax the computable parsimony factor constraint imposed by Mex and investigate whether learning power is thereby enhanced. One way we do so is by allowing lim-computable parsimony factors. It will be convenient to refer sometimes to lim-computable functions as $\lim _{*}$-computable functions - and their formal definition is handled with the next two definitions.

Definition 4 Suppose $h:(N \times N) \rightarrow N$. Then,

$$
\lim _{t \rightarrow \infty} h(x, t) \stackrel{\text { def }}{=} \begin{cases}y, & \text { if }\left(\forall^{\infty} t\right)[h(x, t)=y] ; \\ \uparrow, & \text { otherwise. }\end{cases}
$$

We write $h(x, \infty)$ for $\lim _{t \rightarrow \infty} h(x, t)$.

\section{Definition 5}

(a) $g: N \rightarrow N$ is $\lim _{*}$-computable $\stackrel{\text { def }}{\Leftrightarrow}(\exists$ computable $h:(N \times N) \rightarrow N)(\forall x)[g(x)=h(x, \infty)]$.

(b) $g: N \rightarrow N$ is $\lim _{*}$-computable as witnessed by $h \stackrel{\text { def }}{\Leftrightarrow} h$ computable: $(N \times N) \rightarrow N$ and $(\forall x)[g(x)=h(x, \infty)]$.

Intuitively, in Definition $5, h(x, t)$ is the output at discrete time $t$ of a mind changing algorithm for $g$ (acting on input $x) .(\forall x)[g(x)=h(x, \infty)]$, for $h$ computable, means, then, that, for all $x$, at all but finitely many times $t$, the output of the mind changing algorithm on input $x$ is $g(x)$.

It is easy to show that

$$
(\exists \text { lim-computable } g)(\forall \text { computable } f)\left(\forall^{\infty} x\right)[g(x)>f(x)] .
$$

We can extend Definition 5 to define $\lim _{*}$-computable functions from $N^{n} \rightarrow N$, for $n>1$. We omit the details.

\section{Definition 6}

(a) A learning machine $\mathbf{M} \mathbf{L i m}_{*} \mathbf{M e x}$-identifies $\mathcal{S} \stackrel{\text { def }}{\Leftrightarrow}$ there is a monotonically non-decreasing lim-computable function $g$ such that, $\mathcal{S} \subseteq g$-Mex $(\mathbf{M})$.

(b) $\mathbf{L i m}_{*} \mathbf{M e x}=\left\{\mathcal{S} \mid(\exists \mathbf{M})\left[\mathbf{M} \mathbf{L i m}_{*} \mathbf{M e x}\right.\right.$-identifies $\left.\left.\mathcal{S}\right]\right\}$.

Hence, our definition requires that the machines converge to a program that is not-so-nearlyminimal-size. We mostly write LimMex instead of $\mathbf{L i m}_{*} \mathbf{M e x}$. Chen [Che82] showed that LimMex $\subset$ Ex. From Corollary 35 in Section 3, Mex $\subset$ LimMex.

In Definition 5, the underlying mind-changing algorithm is allowed to change its mind a finite, yet unbounded, number of times. One may, of course, consider the effects of restricting the number of mind changes of such algorithms. 
As noted earlier, in the context of computability-theoretic learning theory, Freivalds and Smith [FS93] first introduced the use of notations for constructive ordinals to bound mind changes.

In this paper, we use notations for constructive ordinals to bound the mind changes in procedures for lim-computable functions. We will formally define, for each notation $u \in O$, the concept of a $\lim _{u^{-}}$computable function below (Definition 10). We will then use (non-decreasing) $\lim _{u^{-}}$ computable functions as parsimony factors.

But first, we present an introduction to $O$, constructive ordinals, and needed associated properties.

\section{3 $O$ and Constructive Ordinals}

We proceed very informally. Some familiarity with a treatment of constructive ordinals such as the ones in [Rog67, Sac90] may be useful to readers of this section.

In Kleene's system $O$ [Kle38, Kle44, Kle55, Rog67, Sac90], $2^{0}$ is (by definition) the notation for the ordinal 0. Successor ordinals are those with an immediate predecessor; for example, 1,2,3, $\omega+$ $1, \ldots$ are successor ordinals with respective immediate predecessors $0,1,2, \omega, \ldots$ If $u$ is a notation for the immediate predecessor of a successor ordinal, then a notation for that successor ordinal is (by definition) $2^{u}$. All other ordinals are limit ordinals; for example, $\omega, \omega+\omega, \ldots$ are limit ordinals. Kleene [Kle38, Kle44, Kle55, Rog67, Sac90] defined a natural partial ordering of notations, $<_{o}$, so that two notations so ordered represent respective ordinals with the second larger than the first. We omit details. Suppose $\varphi_{p}(0), \varphi_{p}(1), \varphi_{p}(2), \ldots$ are each notations in $<_{o}$ order. Suppose that the corresponding ordinals are longer and longer initial segments of some limit ordinal which is their sup. For example, some such $p$ generates the respective notations for $0,1,2, \ldots$ in $<_{o}$ order, and $\omega$ is the sup of this sequence. In general, then, $p$ essentially describes how to build the limit ordinal which is the sup of the ordinals with notations $\varphi_{p}(0), \varphi_{p}(1), \varphi_{p}(2), \ldots$. A notation for this limit ordinal is (by definition) $3 \cdot 5^{p}$. Clearly such limit ordinals have infinitely many such notations, different ones for different generating $p$ 's. Nothing else is a notation. We define ' $x={ }_{o} y$ ' to mean ' $x, y \in O$ and $x=y$ '. As in the literature on constructive ordinals, we use ' $x \leq_{o} y$ ' for ' $x<_{o} y \vee x=_{o} y$ ', ' $x \geq_{o} y$ ' to mean ' $y \leq_{o} x$ and ' $x>_{o} y$ ' to mean ' $y<_{o} x$ '. We also recall the function $|\cdot|_{o}: O \rightarrow$ the set of ordinals, defined as follows [Kle38, Kle55, Rog67, Sac90]

$$
\begin{aligned}
|1|_{o} & =0 ; \\
\left|2^{u}\right|_{o} & =|u|_{o}+1 ; \\
\left|3 \cdot 5^{p}\right|_{o} & =\lim _{n \rightarrow \infty}\left|\varphi_{p}(n)\right|_{o} .
\end{aligned}
$$

For all $x, y \in O$, it is true that, if $x<_{o} y$ then $|x|_{o}<|y|_{o}$. It is also true that, for all $y \in O$, if $|y|_{o}=\beta$, then for every $\alpha<\beta$, there is an $x$ such that $x<_{o} y$ and $|x|_{o}=\beta$. If $u \in O$ and $|u|_{o}=\alpha$, then we say that $u$ is for $\alpha$.

It is useful, while reading this paper, to remember that $|1|_{o}=0,|2|_{o}=1$, and $|4|_{o}=2$. Hence, for example, 4 is, then, the notation for the ordinal 2 , i.e, $\underline{2}=4$.

We shall use the following properties of $<_{o}$ in later proofs.

Fact 7 (Kleene [Kle55]) For all $x, y \in N$,

(a) $x<_{o} y \Rightarrow x \in O \wedge y \in O$.

(b) $x \in O \Rightarrow 1 \leq_{o} x$.

(c) $x<_{o} y \Rightarrow y \neq 1$.

(d) $x<_{o} 2^{y} \Rightarrow x \leq_{o} y$. 
(e) $x<_{o} 3 \cdot 5^{p} \Rightarrow(\exists n)\left[x<_{o} \varphi_{p}(n)\right]$.

(f) $x \leq_{o} z \wedge y \leq_{o} z \Rightarrow x<_{o} y \vee x=_{o} y \vee x>_{o} y$.

The following fact about notations will also be used.

Fact 8 (Rogers [Rog67], Sacks [Sac90]) There exist computable functions $h_{1}$ and $h_{2}$ such that, for all $v \in O$,

(a) $W_{h_{1}(v)}=\left\{u \mid u<_{o} v\right\}$;

(b) $W_{h_{2}(v)}=\left\{\left\langle u_{1}, u_{2}\right\rangle \mid u_{1}<_{o} u_{2}<_{o} v\right\}$ is a well-ordering isomorphic to $|v|_{o}$.

\section{$2.4 \quad$ O-Countdown Functions and Learning Criteria}

Definition 9 A computable mapping $\mathbf{F}:(N \times N) \rightarrow O$ is an $O$-countdown function $\stackrel{\text { def }}{\Leftrightarrow}$ for all $x, t, \mathbf{F}(x, t+1) \leq_{o} \mathbf{F}(x, t)$.

Intuitively, $h$ in Definition 10 just below plays a similar role to $h$ in Definition 5 , and the function F in Definition 10 serves as a "transfinite" counter that counts down from a preset value. As before, $t$ can be thought of a discrete time paramenter. Further explanation is given just after Definition 10.

Definition 10 Suppose $u \in O . g: N \rightarrow N$ is $\lim _{u}$-computable $\stackrel{\text { def }}{\Leftrightarrow}$ there exists a computable function $h:(N \times N) \rightarrow N$ and an $O$-countdown function $\mathbf{F}$, such that, for all $x$ and $t$,

(a) $g(x)=h(x, \infty)$,

(b) $\mathbf{F}(x, 0) \leq_{o} u$, and

(c) $h(x, t+1) \neq h(x, t) \Rightarrow \mathbf{F}(x, t+1)<_{o} \mathbf{F}(x, t)$.

We define lim $_{u}$-computable as witnessed by $\mathbf{F}$ and $h$ along the lines of part (b) of Definition 5; we omit the details.

Part (b) of Definition 10 initializes the transfinite counter at $\leq_{o} u$. From Fact 7 (a), it follows that part (c) restricts the counter values to be notations $\in O$. By Fact 8 , the notations $<_{o} u$ are well-ordered; hence, part (c) also implies that the counter cannot descend infinitely. Part (c) also guarantees that, when $h$ has a mind change, then the counter must decrement. This part does not restrict how much it decrements. It also allows the transfinite counter to decrement without an accompanying mind change in $h$. Note that parts (b) and (c) imply that $h(y, \infty)$ is defined and part (a) defines $g(y)$ to be the value $h(y, \infty)$. Thus, $\mathbf{F}$ acts as a countdown function that starts with a notation in $O$ and then counts down.

In Definition 11 below, we use (non-decreasing) $\lim _{u}$-computable functions as parsimony factors. This is similar to what we did in Definition 6. When $\lim _{u}$-computable functions are so used as parsimony factors, we shall ofter refer to them as parsimony factors of order $u$.

\section{Definition 11}

Suppose $u \in O$. Then,

(a) A learning machine $\mathbf{M} \mathbf{L i m}_{u} \mathbf{M e x}$-identifies $\mathcal{S} \stackrel{\text { def }}{\Leftrightarrow}$ there is a monotonically non-decreasing lim $_{u}$-computable function $g$ such that, $\mathcal{S} \subseteq g$-Mex $(\mathbf{M})$.

(b) $\operatorname{Lim}_{u} \mathbf{M e x}=\left\{\mathcal{S} \mid(\exists \mathbf{M})\left[\mathbf{M} \mathbf{L i m}_{u} \mathbf{M e x}\right.\right.$-identifies $\left.\left.\mathcal{S}\right]\right\}$.

In Definition 11 just above, we restrict the parsimony factor, $g$, to be monotonically nondecreasing. The reason is that then (but not otherwise) the $\mathbf{L i m}_{u} \mathbf{M e x}$ criteria are easily shown to be independent of the underlying acceptable system. It even turns out (and is easy to show) that there is a $\lim _{\underline{\underline{1}}}$-computable function $g$ such that, for no $n \in N$ and for $n o \lim _{\underline{\underline{n}}}$-computable, monotonically non-decreasing function $g^{\prime}$ do we have that $g^{\prime}$ dominates $g$. 


\subsection{Basic Properties of $O$-Notations}

We need the results of this section regarding addition, multiplication and exponentiation of ordinal notations; as noted above, these operations are denoted $+_{o}, \times_{o}$ and $\{\cdot\}_{o}$ respectively. Recall from Section 1.2 above that our $+_{o}$ and $\times_{o}$ feature extra base cases over what would be needed merely to get the embedding into the corresponding ordinal operations and that these extra base cases are to ensure some additional technically useful properties.

In unparenthesized expressions involving these operations, $\left\{\cdot{ }^{\circ}\right\}_{o}$ has higher priority than $\times_{o}$ which in turn has higher priority than $+_{o} \cdot{ }^{18}$

Note 12 For these theorems, it is useful to recall that 1 is a notation for 0 and that 2 is a notation for 1 (i.e., $|1|_{o}=0,|2|_{o}=1$ ) and that 0 and 7 are not notations (for any ordinal) in the $O$ system of notations.

Theorem 13 There exists a computable function $+_{o}$ such that, for all $x, y \in N$,

$$
x+{ }_{o} y= \begin{cases}y, & \text { if } x=1 ;(\text { clause i) } \\ x, & \text { if } y=1 \wedge x \neq 0 \wedge x \neq 1 ; \text { (clause ii) } \\ 2^{(x+o{ } m)}, & \text { if } y=2^{m} \wedge m \neq 0 \wedge x \neq 1 ; \text { (clause iii) } \\ 3 \cdot 5^{q}, & \text { if } y=3 \cdot 5^{p} \wedge x \neq 1,(\text { clause iv) } \\ & \left.\quad \text { where }(\forall n)\left[\varphi_{q}(n)=x+\varphi_{o} \varphi_{p}(n)\right]\right) ; \\ 7, & \text { otherwise. }(\text { clause } \mathrm{v})\end{cases}
$$

Furthermore, $q$ in Clause (iv) just above is a computable, 1-1 function of $x$ and $p$.

PROOF.

The proof is an easy modification of that of [Rog67, Theorem XVII, Chapter 11, pages 209-210] and uses the 1-1 Parametric Recursion Theorem. ${ }^{19}$

The 1-1-ness of $q$ in Theorem 13 is crucial for proving the right-to-left implication of part (e) of Theorem 14, which in turn is necessary for proving many results that follow.

The just above theorem is a modification of a theorem in [Kle55]. The first Clause (clause (i)) has been added in order to make $+_{o}$ have the useful property: for all $y, 1+_{o} y=y$ (i.e., for all $y, \underline{0}+_{o} y=y$ ), which is not true without the special handling of the $x=1$ case. $^{20}$ This property is used, for example, in the proof of Lemma 37. As in [Kle55], we treat the $x=0$ case specially (doing so helps to ensure that $0+_{o} 2=2^{7}$ (and not 1), which is essential in proving the right-to-left side of Theorem 14(a).)

Theorem 14 For all $x, y$ and $z \in N$,

(a) $x, y \in O \Leftrightarrow x++_{o} y \in O$.

(b) $x, y \in O \Rightarrow\left|x+_{o} y\right|_{o}=|x|_{o}+|y|_{o}$.

(c) $x, y \in O \wedge y \neq 1 \Rightarrow x<_{o} x++_{o} y$.

(d) $x, y \in O \wedge z<_{o} y \Leftrightarrow x+_{o} z<_{o} x+_{o} y$.

(e) $x \in O \wedge z={ }_{o} y \Leftrightarrow x+_{o} z={ }_{o} x+{ }_{o} y$.

(f) $y \in O \wedge x \leq_{o} z<_{o} x+_{o} y \Leftrightarrow\left(\exists y^{\prime}\right)\left[y^{\prime}<_{o} y \wedge x+_{o} y^{\prime}=_{o} z\right]$.

Furthermore, in the left-to-right implication of $(f), y^{\prime}$ is unique.

\footnotetext{
${ }^{18}$ Also, we assume that each of these operators has higher priority than $<_{o}, \leq_{o},>_{o}, \ldots$, which have higher priority than $\in,=, \neq$, which have higher priority than $\wedge$ and $\vee$, which in turn have higher priority than $\Rightarrow$ and $\Leftrightarrow$.

${ }^{19}$ Our extra base case causes no subtleties with modifying Rogers' construction.

${ }^{20}$ Recall from Note 12,1 is the notation for the ordinal 0.
} 
ProOF.

The proof is straightforward, but tedious, and involves Fact 7(a-e) and careful application of transfinite induction on notations. ${ }^{21}$

We note that, similar to the case of + for ordinals, $+_{o}$ for notations is non-commutative; $+_{o}$ is, however, also non-associative unlike + for ordinals. We adopt the convention that $x+{ }_{o} y+_{o} z$ means $\left(x+{ }_{o} y\right)+_{o} z$. The non-associativity of $+_{o}$ leads to some subtleties while working with notations, that are otherwise absent when working with ordinals.

We next define $\times_{o}{ }^{22}$

Theorem 15 There is a computable function $\times_{o}$ such that, for all $x, y \in N$,

$$
x \times_{o} y= \begin{cases}1, & \text { if } y=1 \vee x=1 ; \text { (clause i) } \\ y, & \text { if } x=2 \wedge y \neq 1 ;(\text { clause ii) } \\ \left(x \times_{o} m\right)+_{o} x, & \text { if } y=2^{m \wedge} \wedge \neq \wedge \\ 3 \cdot 5^{q}, & x \neq 1 \wedge x \neq 2 ;(\text { clause iii) } \\ & \text { if } y=3 \cdot 5^{p} \wedge x \neq 1 \wedge x \neq 2,(\text { clause iv) } \\ 7, & \left.\quad \text { otherwise. }(\forall n)\left[\varphi_{q}(n)=x \times_{o} \varphi_{p}(n)\right]\right)\end{cases}
$$

Furthermore, $q$ as in Clause (iv) is a computable, 1-1 function of $x$ and $p$.

ProOF.

The proof is also similar to that of [Rog67, Theorem XVII, Chapter 11, pages 209-210] (and uses the 1-1 Parametric Recursion Theorem).

The 1-1-ness of $q$ in Theorem 15 is crucial for proving the right-to-left implication of part (e) of Theorem 16, which in turn is necessary for proving many results that follow.

The first two Clauses (clauses (i) and (ii)) in the just above Theorem ensure that useful properties of $\times_{o}$ hold. For example: (a) for all $y, 1 \times_{o} y=1$ (i.e., for all $y, \underline{0} \times_{o} y=\underline{0}$ ); (b) for all $y$, $2 \times_{o} y=y$ (i.e., for all $y, \underline{1} \times_{o} y=y$ ). Property (b) just listed, is essential, for example, in proving Lemma 25 presented further below.

Similar to the case of $\times$ (usually written $\cdot$ ) for ordinals, $\times_{o}$ for notations is not commutative; however, $\times$ for ordinals is associative, but $\times_{o}$ for notations is not. As for $+_{o}$, in any unparenthesized expressions involving $\times_{o}$, we associate to the left.

Analogous to Theorem 14, we have the following theorem for $\times_{o}$.

Theorem 16 For all $x, y$ and $z \in N$,

(a) $x, y \in O \vee x=1 \vee y=1 \Leftrightarrow x \times_{o} y \in O$.

(b) $x, y \in O \Rightarrow\left|x \times_{o} y\right|_{o}=|x|_{o} \times|y|_{o}$.

(c) $x>_{o} 1 \wedge y>_{o} 2 \Rightarrow x<_{o} x \times_{o} y$.

(d) $x>_{o} 1 \wedge y \in O \wedge z<_{o} y \Leftrightarrow x \times_{o} z<_{o} x \times_{o} y$.

(e) $x=1 \vee\left(x \in O \wedge z=_{o} y\right) \Leftrightarrow x \times_{o} z==_{o} x \times_{o} y$.

$(f) y \in O \wedge x \leq_{o} z<_{o} x \times_{o} y \Leftrightarrow\left(\exists x^{\prime}<_{o} x\right)\left(\exists y^{\prime} \mid 1<_{o} y^{\prime}<_{o} y\right)\left[z={ }_{o}\left(x \times_{o} y^{\prime}\right)+_{o} x^{\prime}\right]$.

Furthermore, in $(f) \Rightarrow, x^{\prime}$ and $y^{\prime}$ are both unique.

\footnotetext{
${ }^{21}$ An example of a proof by transfinite induction is part of the proof of Theorem 36. See [Kle44, Kle55, Sac90] for other examples of such inductions.

${ }^{22}$ See Note 12 regarding the notations $1,2 \in O$.
} 
ProOF.

As for $+_{o}$, the proof is straightforward, but tedious, and involves Fact 7(a-e), Theorem 14(a-f) and careful application of transfinite induction on notations.

Finally, we define exponentiation for notations in $O$ as follows. ${ }^{23}$

Theorem 17 There is a computable function $\{\cdot\}_{o}$ such that, for all $x, y \in N$,

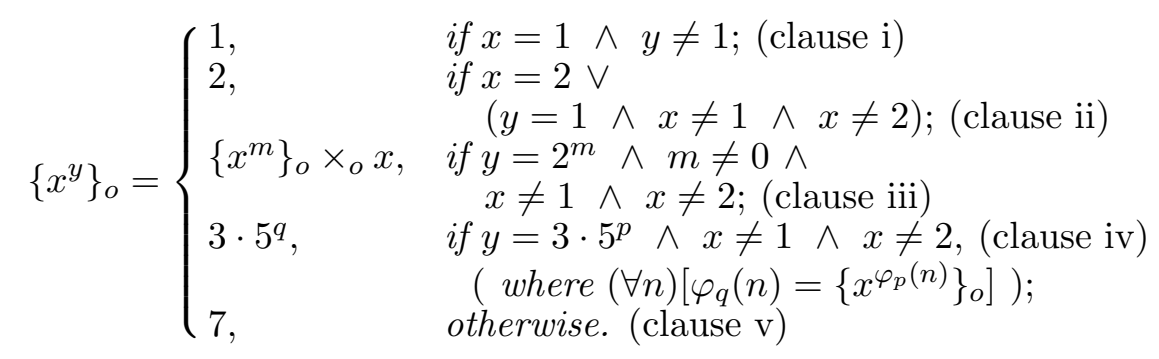

Furthermore, $q$ as in Clause (iv), is a computable, 1-1 function of $x$ and $p$.

PROOF.

The proof is again similar to that of [Rog67, Theorem XVII, Chapter 11, pages 209-210] (and uses the 1-1 Parametric Recursion Theorem).

The 1-1-ness of $q$ in Theorem 17 is crucial for proving the right-to-left implication of part (e) of Theorem 18 .

Similar to exponentiation for ordinals, $\left\{\cdot^{\cdot}\right\}_{0}$ for notations is neither commutative nor associative. We note that, in general, for $x, y \in N,\left\{x^{y}\right\}_{o}$ is quite different from $x^{y}$.

The following are some useful properties of $\{\cdot\}_{0}$.

Theorem 18 For all $x, y$ and $z \in N$,

(a) $x, y \in O \wedge(x \neq 1 \vee y \neq 1) \Rightarrow\left\{x^{y}\right\}_{o} \in O$.

(b) $x, y \in O \wedge(x \neq 1 \vee y \neq 1) \Rightarrow\left|\left\{x^{y}\right\}_{o}\right|_{o}=|x|_{o}^{|y|_{o}}$.

(c) $x>_{o} 2 \wedge y>_{o} 2 \Rightarrow x<_{o}\left\{x^{y}\right\}_{o}$.

(d) $x>_{o} 2 \wedge z<_{o} y \Rightarrow\left\{x^{z}\right\}_{o}<_{o}\left\{x^{y}\right\}_{o}$.

(e) $x>_{o} 2 \wedge z=y \in O \Rightarrow\left\{x^{z}\right\}_{o}=\left\{x^{y}\right\}_{o} \in O$.

(f) $x>_{o} 1 \wedge x^{\prime}<_{o} x \wedge y \in O \Rightarrow\left\{x^{y}\right\}_{o} \times_{o} x^{\prime}<_{o}\left\{x^{(y+o 2)}\right\}_{o}$.

ProOF.

As for $+_{o}$ and $\times_{o}$, the proof is straightforward, but tedious, and involves Fact 7(b-e), Fact 8(b), Theorem 16(a-d) and careful application of transfinite induction on notations.

We note that, with modifications for special cases, the right-to-left implications of parts (a), (d), (e) and (f) of Theorem 18 are true, but not necessary for this paper. So also is a property similar to Theorem 16(f).

\footnotetext{
${ }^{23}$ Again, see Note 12 regarding the notations $1,2 \in O$.
} 


\section{Main Results}

We recall that by Convention 1 , for $n \in N, \underline{n}$ is the unique notation in $O$ for $n$. Clearly, we have

Proposition 19 For all $u, v \in O$ such that $u \leq_{o} v$,

$$
\operatorname{Mex}=\operatorname{Lim}_{\underline{0}} \operatorname{Mex} \subseteq \operatorname{Lim}_{u} \operatorname{Mex} \subseteq \operatorname{Lim}_{v} \operatorname{Mex} \subseteq \operatorname{LimMex} \subseteq \mathbf{E x} .
$$

The next Theorem shows that one does not always get increased learning power by using parsimony factors of greater orders. It shows more specifically that, when boosting the order $u$ to any order strictly $<_{o}$ the order obtained by multiplying (on the right, with $\times_{o}$ ) $u$ by some $w$ for $\omega$, we get collapse of the corresponding parsimony restricted criteria.

Theorem 20 For all $u, u^{\prime} \in O$ and notations $w$ for $\omega$, such that $u \leq_{o} u^{\prime}<_{o} u \times_{o} w$, $\mathbf{L i m}_{u^{\prime}} \mathbf{M e x}=$ $\operatorname{Lim}_{u}$ Mex.

Proof of TheOrem 20. Follows from

Lemma 21 For all $u, u^{\prime} \in O$ and all notations $w$ for $\omega$ such that $u^{\prime}<_{o} u \times_{o} w$, every monotonically non-decreasing lim $_{u^{\prime}}$-computable function is dominated by a monotonically non-decreasing lim $_{u^{-}}$ computable function.

Proof of Lemma 21. Let $u, u^{\prime}, w \in O$, where $|w|_{o}=\omega$, be such that $u^{\prime}<_{o} u \times_{o} w$. Using the definition of $\times_{o}$ and Fact $7(\mathrm{e})$, there exists $k \in N$ such that $u^{\prime}<_{o} u \times_{o} \underline{k}$. Clearly, such a $k$ must be greater than 0 . Hence, it suffices to prove that for each $k>0$, every monotonically non-decreasing $\lim _{u \times_{o} \underline{k}}$-computable function is dominated by a monotonically non-decreasing $\lim _{u}$-computable function.

Therefore, suppose $k>0$ and $g$ is a monotonically non-decreasing $\lim _{u \times_{o} \underline{\underline{k}}}$-computable function as witnessed by $h$ and $\mathbf{F}$. Therefore, for all $x, \mathbf{F}(x, 0) \leq_{o} u \times_{o} \underline{k}$. Let $k^{\prime}$ be the largest integer, $0 \leq k^{\prime} \leq k$, such that, for all but finitely many $x, \mathbf{F}(x, \infty) \geq_{o} u \times_{o} \underline{k}^{\prime}$. Let $x_{m}$ be such that, for all $x \geq x_{m}, \mathbf{F}(x, \infty) \geq_{o} u \times_{o} \underline{k}^{\prime}$.

Clearly, by our choice of $k^{\prime}$ and $x_{m}$, there exist infinitely many $x \geq x_{m}$ such that $u \times_{o} \underline{k}^{\prime} \leq_{o}$ $\mathbf{F}(x, \infty)<_{o} u \times_{o} \underline{k^{\prime}+1}$. Also, using Fact 8(a), $X=\left\{x \geq x_{m} \mid u \times_{o} \underline{k^{\prime}} \leq_{o} \mathbf{F}(x, \infty)<_{o} u \times_{o} \underline{k^{\prime}+1}\right\}$ is computably enumerable. Let $Y=\left\{y_{0}<y_{1}<\ldots\right\}$ be an infinite computable subset of $X$.

We now define $h^{\prime}, \mathbf{F}^{\prime}$ as follows.

For all $x$, let $h^{\prime}(x, 0)=h\left(y_{x}, t^{\prime}\right)$, where $t^{\prime}$ is the least number such that $\mathbf{F}\left(y_{x}, t^{\prime}\right)<_{o} u \times_{o} \underline{k^{\prime}+1}$; for $t>0, h^{\prime}(x, t)=h\left(y_{x}, t^{\prime}+t\right)$.

For all $x$, let $\mathbf{F}^{\prime}(x, t)=u^{\prime}$, where $\mathbf{F}\left(y_{x}, t^{\prime}+t\right)=u \times_{o} \underline{k}^{\prime}+_{o} u^{\prime}\left(u^{\prime}\right.$ can be effectively found as follows: since $u \times_{o} \underline{k^{\prime}} \leq_{o} \mathbf{F}\left(y_{x}, t^{\prime}+t\right)<_{o} u \times_{o} \underline{k^{\prime}}+_{o} u$, by Theorem 14(f), $\left(\exists ! u^{\prime}<_{o} u\right)\left[\mathbf{F}\left(y_{x}, t^{\prime}+t\right)=u \times_{o} \underline{k^{\prime}}+_{o} u^{\prime}\right]$; then, using Fact 8(a), we can effectively find this $u^{\prime}$ ).

Let $g^{\prime}(x)=h^{\prime}(x, \infty)$. It is easy to verify that for all $x, g^{\prime}(x) \geq g(x)$, and $g^{\prime}$ is monotonically non-decreasing, and that $h^{\prime}$ and $\mathbf{F}^{\prime}$ witness that $g^{\prime}$ is $\lim _{u}$-computable.

(LEMMA 21)

Clearly, the theorem follows from Lemma 21.

(THEOREM 20)

When $u$ is a notation for a finite ordinal, we can get the following result.

Lemma 22 For all $n \in N$, every monotonically non-decreasing lim $_{\underline{n}}$-computable function is dominated by a monotonically non-decreasing computable function. 
ProOF.

From Lemma 21, we get that for every $n>0$, every monotonically non-decreasing $\lim _{\underline{n}^{-}}$ computable function is dominated by a monotonically non-decreasing $\lim _{\underline{1}}$-computable function. Hence, it is sufficient to prove that every monotonically non-decreasing $\lim _{\underline{1}}$-computable function is dominated by a monotonically non-decreasing computable function.

Therefore, suppose $g$ is a monotonically non-decreasing $\lim _{\underline{1}}$-computable function as witnessed by $h$ and $\mathbf{F}$. Hence, for each $x$, there exists at most one $t$ such that $h(x, t) \neq h(x, t+1)$.

Case 1: $\left(\forall^{\infty} x\right)(\forall t)[h(x, t)=h(x, t+1)]$.

In this case, let $x_{0}$ be such that $\left(\forall x \geq x_{0}\right)(\forall t)[h(x, t)=h(x, t+1)]$. Define $g^{\prime}$ as follows: for all $x, g^{\prime}(x)=h\left(x_{0}+x, 0\right)$.

Case 2:. Not Case 1.

Let $X=\{x \mid(\exists t)[h(x, t) \neq h(x, t+1)]\}$. Let $Y=\left\{y_{0}<y_{1}<\ldots\right\}$ be an infinite computable subset of $X$. Define $g^{\prime}$ as follows: for all $x, g^{\prime}(x)=h\left(y_{x}, t+1\right)$, where $t$ is such that $h\left(y_{x}, t\right) \neq$ $h\left(y_{x}, t+1\right)$; clearly, by the properties of $h$ and $Y$, there is exactly one such $t$ for each $y \in Y$.

Also, in both the above cases, $g^{\prime}$ is computable, monotonically non-decreasing and dominates $g$.

Hence, we get

Theorem 23 For all $n \in N, \operatorname{Lim}_{\underline{n}} \operatorname{Mex}=$ Mex.

One of our main results, our Strong Hierarchy Theorem (Theorem 40 below), shows that the collapses of the previous two theorems are optimal for parsimony orders along naturally associated $<_{o}$-paths. Before we present our Strong Hierarchy Theorem, we present another of our main results, our General Hierarchy Theorem, as the next theorem (Theorem 32 below). To do this, we require some preliminary results.

As noted earlier, parsimony factors are always monotonically non-decreasing ( $\lim _{u}$-computable) functions. In constructions below, we sometimes first define $\lim _{u}$-computable functions that are not necessarily monotonically non-decreasing, before we define appropriate parsimony factors that dominate them. Lemma 24 below gives us useful sufficient conditions to determine the orders of so obtained dominating parsimony factors.

Lemma 24 Suppose $u \in O$. Suppose $\left\{\psi_{n} \mid n \in N\right\}$ is a family of (partial) computable functions, where each $\psi_{n}$ is a function from $N^{n+1}$ to $N$ (which are defined when all their arguments $\leq_{o} u$ ), with the following three properties:

(1) For $u_{n}, \ldots, u_{0} \leq_{o} u, \psi_{n}\left(u_{n}, \ldots, u_{0}\right)$ can be obtained effectively from $n$ and $u_{n}, \ldots, u_{0}$.

(2) For each $n, \psi_{n}$ is strictly monotonically increasing (with respect to $\leq_{o}$ ) on each of its arguments (as long as its arguments are $\leq_{o} u$ ).

(3) For each $n, \psi_{n}(u, \ldots, u)<_{o} \psi_{n+1}(u, \ldots, u)$.

Let $v$ be a notation for $\lim _{n \rightarrow \infty}\left|\psi_{n}(u, \ldots, u)\right|_{o}$, obtained using any computable procedure that generates, in increasing order w.r.t $<_{o}$, an infinite subset of $\left\{z \leq_{o} \psi_{n}(u, \ldots, u) \mid n \in N\right\}$. Then, every lim $_{u}$-computable function $f$ is dominated by a monotonically non-decreasing $\lim _{v}$-computable function $f^{\prime}$ (i.e., for all $x, f(x) \leq f^{\prime}(x)$ ).

Proof. Suppose the hypotheses. Suppose $f$ is $\lim _{u}$-computable as witnessed by $h$ and $\mathbf{F}$. We will define a function $f^{\prime}$ bounding $f$, which is $\lim _{v}$-computable as witnessed by $h^{\prime}$ and $\mathbf{F}^{\prime}$ defined below. 
Let

$$
\begin{aligned}
h^{\prime}(x, t) & =\max \left(\left\{h\left(x^{\prime}, t\right) \mid x^{\prime} \leq x\right\}\right) \\
\mathbf{F}^{\prime}(x, t) & =\psi_{x+1}(\mathbf{F}(0, t), \mathbf{F}(1, t), \ldots, \mathbf{F}(x, t)) \\
f^{\prime}(x) & =h^{\prime}(x, \infty) .
\end{aligned}
$$

It is easy to verify that $f^{\prime}, h^{\prime}, \mathbf{F}^{\prime}$ satisfy the properties as claimed.

Lemma 25 Suppose $u, w \in O$ and $w$ is for $\omega$. Then, for each lim $_{u}$-computable $g$, there exists a monotonically non-decreasing $\lim _{\left\{(u+o \underline{1})^{w}\right\}_{o}}$-computable $g^{\prime}$, such that $g^{\prime}$ dominates $g$.

Proof of Lemma 25.

If $g$ is a $\lim _{\underline{0}}$-computable function, then it is clearly computable. Define $g^{\prime}(x)=\max (\{g(i) \mid$ $i \leq x\}$ ). Clearly, $g^{\prime}$ is computable, monotonically non-decreasing, and dominates $g$. Also, for all $w$ for $\omega,\left\{\left(\underline{0}+_{o} \underline{1}\right)^{w}\right\}_{o}=\underline{1}$. Since every computable function is also $\lim _{\underline{1}}$-computable, the lemma holds for $u=\underline{0}$.

For the $u>\underline{0}$ cases, we will use Lemma 24. Let $\psi_{n}$ be defined recursively as follows (Note: we are only interested in defining $\psi_{n}$ on arguments $\leq_{o} u$; by Fact $8(\mathrm{a})$, doing so is algorithmically possible.)

Firstly, for all $i \in N$, let

$$
v_{i}=\left\{\left(u+{ }_{o} \underline{1}\right)^{\underline{i}}\right\}_{o}
$$

For all $u_{0} \leq_{o} u$, let

$$
\psi_{0}\left(u_{0}\right) \stackrel{\text { def }}{=} u_{0} .
$$

For all $n>0$, for all $u_{0}, \ldots, u_{n} \leq_{o} u$, let

$$
\psi_{n}\left(u_{n}, \ldots, u_{0}\right) \stackrel{\text { def }}{=}\left(v_{n} \times_{o} u_{n}\right)+_{o} \psi_{n-1}\left(u_{n-1}, \ldots, u_{0}\right)
$$

We note that $\psi_{n}\left(u_{n}, \ldots, u_{0}\right)$ is just the summation of $v_{n} \times_{o} u_{n}, \ldots, v_{1} \times_{o} u_{1}, u_{0}$ in a right associative manner. Clearly, $\left\{\psi_{n} \mid n \in N\right\}$ satisfies property (1) of Lemma 24.

Claim 26 For all $n$, for all $u_{0}, \ldots, u_{n} \leq_{o} u$, we have that $\psi_{n}\left(u_{n}, \ldots, u_{0}\right)<_{o} v_{n+1}$.

Proof of Claim 26.

We prove this Claim by induction on $n$.

Base Case: $n=0$.

$\psi_{0}\left(u_{0}\right)=u_{0}$. Also, $v_{1}=\left\{(u+o \underline{1})^{\underline{1}}\right\}_{o}=u+_{o} \underline{1}$. Since $u_{0} \leq_{o} u$, we get $\psi_{0}\left(u_{0}\right)<_{o} v_{1}$.

Inductive Case: Suppose the claim is true for $n=k$ (Inductive Hypothesis (IH)).

We will show that it holds for $n=k+1$.

$\psi_{k+1}\left(u_{k+1}, \ldots, u_{0}\right)=v_{k+1} \times_{o} u_{k+1}+_{o} \psi_{k}\left(u_{k}, \ldots, u_{0}\right)<_{o}$ (by IH) $v_{k+1} \times_{o} u_{k+1}+_{o} v_{k+1}=($ by Theorems 13 and 15) $v_{k+1} \times_{o}\left(u_{k+1}+_{o} \underline{1}\right)=\left\{\left(u+_{o} \underline{1}\right) \frac{k+1}{\underline{k}}\right\}_{o} \times_{o}\left(u_{k+1}+_{o} \underline{1}\right)$; also, since $u_{k+1} \leq_{o}$ $u$, we get $^{24}$ that $u_{k+1} t_{o} \underline{1} \leq_{o} u+_{o} \underline{1}$, and then, by parts (d) and (e) of Theorem 16, we get $\left\{(u+o \underline{1}) \frac{k+1}{2}\right\}_{o} \times_{o}\left(u_{k+1}+{ }_{o} \underline{1}\right) \leq_{o}\left\{(u+o \underline{1}) \frac{k+1}{}\right\}_{o} \times_{o}(u+o \underline{1})=\left(\right.$ by Theorem 17) $\left\{(u+o \underline{1}) \frac{k+2}{\square}\right\}_{o}=v_{k+2}$.

From Claim 26 and the definitions of $\psi_{n}$ 's, we get for all $n, \psi_{n}(u, \ldots, u)<_{o} v_{n+1} \leq_{o}$ (since $\left.u>_{o} \underline{0}\right) \psi_{n+1}(u, \ldots, u)$. Hence, $\left\{\psi_{n} \mid n \in N\right\}$ satisfies property (3) of Lemma 24 .

\footnotetext{
${ }^{24}$ Proof: If $u_{k+1}=u$, then $u_{k+1}+_{o} \underline{1}=2^{u_{k+1}}=2^{u}=u+{ }_{o} \underline{1}$; otherwise, if $u_{k+1}<_{o} u$, then using the contrapositive of Fact $7(\mathrm{~d})$, we get $u_{k+1} t_{o} \underline{1} \leq_{o} u$, which, by Theorem $14(\overline{\mathrm{c}})$, is $<_{o} u t_{o} \underline{1}$. Therefore, $u_{k+1} t_{o} \underline{1} \leq_{o} u+_{o} \underline{1}$.
} 
Claim 27 Suppose $n, u_{n}, \ldots, u_{0}$ and $u_{n}^{\prime}, \ldots, u_{0}^{\prime}$ are given such that for $i \leq n$, each $u_{i}, u_{i}^{\prime} \leq_{o} u$, and, there exists a $j \leq n$, such that

(a) for all $j^{\prime}, j<j^{\prime} \leq n, u_{j^{\prime}}=u_{j^{\prime}}^{\prime}$.

(b) $u_{j}<_{o} u_{j}^{\prime}$.

Then $\psi_{n}\left(u_{n}, \ldots, u_{0}\right)<_{o} \psi_{n}\left(u_{n}^{\prime}, \ldots, u_{0}^{\prime}\right)$.

Proof of Claim 27. Suppose the hypotheses.

To show the claim, it suffices to show that for all $j \leq n, \psi_{j}\left(u_{j}, \ldots, u_{0}\right)<_{o}\left(v_{j} \times_{o} u_{j}^{\prime}\right)$. For then, since $u_{j}=u_{j}^{\prime}$ for all $j^{\prime}$ such that $j<j^{\prime} \leq n$, we get

$$
\begin{aligned}
\psi_{n}\left(u_{n}^{\prime}, \ldots, u_{0}^{\prime}\right)={ }_{o} & \left(\left(v_{n} \times_{o} u_{n}^{\prime}\right)+_{o}\left(\ldots+_{o}\left(\left(v_{j+1} \times_{o} u_{j+1}^{\prime}\right)+_{o}\left(v_{j} \times_{o} u_{j}^{\prime}\right)\right) \ldots\right)\right) \\
=_{o} & \left(\left(v_{n} \times_{o} u_{n}\right)+_{o}\left(\ldots+_{o}\left(\left(v_{j+1} \times_{o} u_{j+1}\right)+_{o}\left(v_{j} \times_{o} u_{j}^{\prime}\right)\right) \ldots\right)\right) \\
>_{o} & \left(\left(v_{n} \times_{o} u_{n}\right)+_{o}\left(\ldots+_{o}\left(\left(v_{j+1} \times_{o} u_{j+1}\right)+_{o} \psi_{j}\left(u_{j}, \ldots, u_{0}\right)\right) \ldots\right)\right) \\
={ }_{o} & \psi_{n}\left(u_{n}, \ldots, u_{0}\right) .
\end{aligned}
$$

We proceed with the proof of Claim 27. If $j=0$, then $\psi_{0}\left(u_{0}\right)=u_{0}<_{o} u_{0}^{\prime}$. Also, by Theorem 17 we have that $v_{0}=\underline{1}$. Furthermore, since we ensured that for all $y, \underline{1} \times_{o} y=y$ (as noted a few paragraphs after Theorem 15), we get $u_{0}^{\prime}=\left(v_{0} \times_{o} u_{0}^{\prime}\right)$. Hence, $\psi_{0}\left(u_{0}\right)<_{o}\left(v_{0} \times_{o} u_{0}^{\prime}\right)$.

If $j>0$, then we have

$$
\begin{aligned}
\psi_{j}\left(u_{j}, \ldots, u_{0}\right) & =_{o} \quad\left(v_{j} \times_{o} u_{j}\right)+_{o} \psi_{j-1}\left(u_{j-1}, \ldots, u_{0}\right) \\
<_{o} & \left(v_{j} \times_{o} u_{j}\right)+_{o} v_{j} \\
=_{o} & \left(v_{j} \times_{o}\left(u_{j}+o \underline{1}\right)\right) \\
\leq_{o} & \left(v_{j} \times_{o} u_{j}^{\prime}\right) .
\end{aligned}
$$

(by Fact 7(d), Theorem 16)

(Claim 27)

Thus $\psi_{n}$ is monotonically increasing in each of its arguments (as long as they are $\leq_{o} u$ ). Hence, property (2) of Lemma 24 is satisfied by $\left\{\psi_{n} \mid n \in N\right\}$. We note that, for all $n$, $\psi_{n}(u, \ldots, u)<_{o}\left\{\left(u+_{o} \underline{1}\right)^{n+1}\right\}_{o}<_{o}$ (by Theorem 18(d)) $\left\{\left(u+_{o} \underline{1}\right)^{w}\right\}_{o}$, for any $w$ for $\omega$. Also, for all $n, \lim _{n \rightarrow \infty}\left|\psi_{n}(u, \ldots, u)\right|_{o}=\left(\left|u+_{o} \underline{1}\right|_{o}\right)^{\omega}$. Hence, taking $v=\left\{\left(u++_{o} \underline{1}\right)^{w}\right\}_{o}$ in Lemma 24, Lemma 25 follows.

(LEMMA 25)

We next define, for each $u \in O$, a useful, self-referential class of computable functions, $\mathcal{C}_{u}$, that helps to establish our Hierarchy Theorems: $\mathcal{C}_{u}$ is learnable with parsimony factors of order $\left\{(u+o \underline{2})^{w}\right\}_{o}$, for any $w$ for $\omega$ (Lemma 30); however, $\mathcal{C}_{u}$ is not learnable with parsimony factors of order $u$ (Lemma 31).

We recall that $\langle\cdot, \cdot, \cdot, \cdot \cdot\rangle$ denotes a computable bijection from $N \times N \times N \times N$ onto $N$ and that $\pi_{i}^{4}, 1 \leq i \leq 4$, denote the corresponding projection functions (i.e $\left.\pi_{i}^{4}\left(\left\langle x_{1}, x_{2}, x_{3}, x_{4}\right\rangle\right)=x_{i}\right)$.

Definition 28 Suppose $u \in O$. Then,

$\mathcal{C}_{u}=\{f \mid$ for some $p$
(a) $\lim _{x \rightarrow \infty} \pi_{1}^{4}(f(x)) \downarrow=p \wedge \varphi_{p}=f$, and
(b) $\lim _{x \rightarrow \infty} \pi_{2}^{4}(f(x)) \downarrow \geq p$, and
(c) $\pi_{3}^{4}(f(0)) \leq_{o} u$, and
(d) $(\forall x)\left[\pi_{3}^{4}(f(x+1)) \leq_{o} \pi_{3}^{4}(f(x))\right]$, and
(e) $(\forall x)\left[\pi_{2}^{4}(f(x)) \neq \pi_{2}^{4}(f(x+1)) \Rightarrow \pi_{3}^{4}(f(x+1))<_{o} \pi_{3}^{4}(f(x))\right]$. \} 
It is helpful to note the following facts about the just above definition. Values in the range of each $f \in \mathcal{C}_{u}$ are interpreted as quadruples; on inputs $0,1,2, \ldots$ to $f$, the first elements of these quadruples may vary but they eventually settle down at some number, say $p$; the second elements settle down at a number $\geq p$; the third elements form a non-increasing (w.r.t. $\leq_{o}$ ) chain of notations in $O$ constrained to decrease whenever there is a mind change in the corresponding second elements; finally, the fourth elements of the quadruple are completely unrestricted (which freedom we use in the diagonalization in step 4 of the proof of Lemma 31).

Lemma 29 For every $u \in O$, there exists a lim $_{u+o \underline{1}}$-computable $g$ (which is not necessarily monotonically non-decreasing) and a machine $\mathbf{M}$ such that,

(a) $\mathbf{M} \mathbf{E x}$-identifies $\mathcal{C}_{u}$ and

(b) for all $\varphi_{i} \in \mathcal{C}_{u}, \mathbf{M}\left(\varphi_{i}\right) \leq g(i)$.

Proof. Let $\mathbf{M}$ be defined as follows. $\mathbf{M}(f[0])=0$; for $n>0, \mathbf{M}(f[n])=\pi_{1}^{4}(f(n-1))$.

Let $h$ and $\mathbf{F}$ be defined as follows.

For all $i$, let $h(i, 0)=0, \mathbf{F}(i, 0)=u+{ }_{o} \underline{1}$.

For all $i, t$, we define $h(i, t+1), \mathbf{F}(i, t+1)$ as follows.

begin computation of $h(i, t+1), \mathbf{F}(i, t+1)$.

if $\Phi_{i}(0)>t$

then

Let $h(i, t+1)=h(i, t)$ and $\mathbf{F}(i, t+1)=\mathbf{F}(i, t)$;

else

Let $x$ be the largest value $\leq t$, such that $\left(\forall x^{\prime} \leq x\right)\left[\Phi_{i}(x) \leq t\right]$.

Enumerate $S=\left\{u^{\prime} \mid u^{\prime}<_{o} \mathbf{F}(i, t)\right\}$ for $t$ steps using a fixed enumerator.

(This is possible since $S$ is uniformly c.e. in $\mathbf{F}(i, t)$, by Fact 8(a).)

if $\pi_{3}^{4}\left(\varphi_{i}(x)\right) \in S$ within $t$ steps of the fixed enumeration above

then

else

Let $h(i, t+1)=\pi_{2}^{4}\left(\varphi_{i}(x)\right)$ and $\mathbf{F}(i, t+1)=\pi_{3}^{4}\left(\varphi_{i}(x)\right)$;

endif

Let $h(i, t+1)=h(i, t)$ and $\mathbf{F}(i, t)=\mathbf{F}(i, t)$.

\section{endif}

end computation of $h(i, t+1), \mathbf{F}(i, t+1)$.

Let $g(i)=h(i, \infty)$. It is easy to verify that $g$ and $\mathbf{M}$ satisfy the requirements of the theorem.

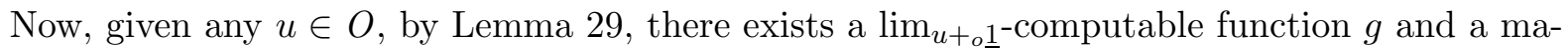
chine $\mathbf{M}$ which $\mathbf{E x}$-identifies $\mathcal{C}_{u}$ such that, for all $i, \varphi_{i} \in \mathcal{C}_{u}$ implies that $\mathbf{M}\left(\varphi_{i}\right) \leq g\left(\operatorname{MinProg}\left(\varphi_{i}\right)\right)$. This $g$ is not necessarily monotonically non-decreasing. However, by noting that the Definition of $+_{o}$ gives $\left(u+_{o} \underline{1}\right)+_{o} \underline{1}=u+_{o} \underline{2}$, and then using Lemma 25, we get that there exists a monotonically

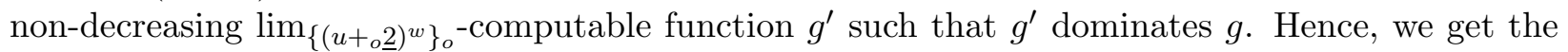
following.

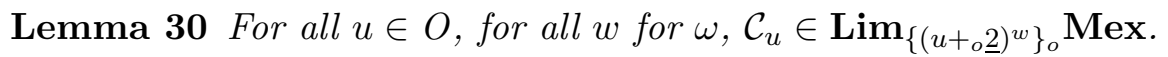


Lemma $31(\forall u \in O)\left[\mathcal{C}_{u} \notin \operatorname{Lim}_{u} \mathbf{M e x}\right]$.

Proof. Suppose by way of contradiction that $\mathcal{C}_{u} \subseteq g$-Mex $(\mathbf{M})$, where $g$ is a monotonically nondecreasing $\lim _{u}$-computable function as witnessed by $h$ and $\mathbf{F}$.

Then by the Operator Recursion Theorem [Cas74, Cas94], there exists a 1-1 increasing function $p$ such that the initial segments (finite or infinite) $\varphi_{p(\cdot)}$ may be defined as follows. Intuitively, while defining these functions, we attempt to define $\varphi_{p(0)}$ such that $\varphi_{p(0)} \in \mathcal{C}_{u}$, and, either (a) $\mathbf{M}\left(\varphi_{p(0)}\right) \downarrow$ and $\operatorname{MinProg}\left(\varphi_{p(0)}\right)$ is such that $g\left(\operatorname{MinProg}\left(\varphi_{p(0)}\right)\right)<\mathbf{M}\left(\varphi_{p(0)}\right)$, or (b) $\mathbf{M}\left(\varphi_{p(0)}\right) \uparrow$; failing this, we will ensure that for some $i>0, \varphi_{p(i)} \in \mathcal{C}_{u}$ and either (a) $\mathbf{M}\left(\varphi_{p(i)}\right) \downarrow$ and $\varphi_{\mathbf{M}\left(\varphi_{p(i)}\right)} \neq \varphi_{p(i)}$, or $\mathbf{M}\left(\varphi_{p(i)}\right) \uparrow$.

The procedure uses global variables cur_bound, cur_notation, cur_maxindex, cur_index, Cancel, and last_mindchange. Then cur_bound $_{s}$, cur_notation ${ }_{s}$, cur_maxindex $_{s}$, cur_index , $_{\text {Cancel }}$, and

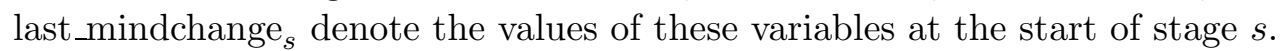

Initially, we let cur_bound ${ }_{0}=h(p(0), 0)$, cur_notation ${ }_{0}=u$, cur_maxindex ${ }_{0}=$ cur_bound $_{0}+2$. cur_index ${ }_{0}=1$. Cancel ${ }_{0}=\emptyset$, last_mindchange ${ }_{0}=0$.

$x_{s}$ denotes the least $x$ such that $\varphi_{p(0)}(x)$ has not been defined at the start of stage $s$. Thus $x_{0}=0$. Go to stage 0 .

Begin stage $s$.

1. For $x<x_{s}$, let $\varphi_{p\left(\operatorname{cur}_{\text {index }}\right)}(x)=\varphi_{p(0)}(x)$.

2. Let $y=x_{s}$.

repeat

2.1. Let $\varphi_{p\left(\text { cur_index }_{s}\right)}(y)=\left\langle p\left(\right.\right.$ cur_index $\left._{s}\right), p\left(\right.$ cur_maxindex $\left._{s}\right)$, cur_notation $\left.{ }_{s}, 0\right\rangle$.

2.2. If $h(p(0)$, last mindchange $s) \neq h(p(0), y)$, then go to step 3 .

2.3. If there exists $i \leq$ cur_bound $_{s}, i \notin$ Cancel $_{s}$, and $z$, such that $x_{s}<z \leq y$ and $\Phi_{i}(z) \leq y$, then go to step 4 .

2.4. If $\mathbf{M}\left(\varphi_{p\left(\text { cur_index }_{s}\right)}[y+1]\right)>$ cur_bound $_{s}$, then go to step 5 .

2.5. Let $y=y+1$.

forever

3. For $x \leq y$, let $\varphi_{p(0)}(x)=\varphi_{p\left(\operatorname{cur}_{-} \text {index }_{s}\right)}(x)$.

Decrement notation: cur_notation ${ }_{s+1}=\mathbf{F}(p(0), y)$.

Change current bound: cur_bound ${ }_{s+1}=h(p(0), y)$.

Change current maximum index: cur_maxindex ${ }_{s+1}=$ cur_maxindex $_{s}+$ cur_bound $_{s+1}+2$.

Record where the last mind change happened: last_mindchange ${ }_{s+1}=y$.

Carry forward unchanged all other global variables (cur_index and Cancel).

Go to stage $s+1$. (Therefore $x_{s+1}=y+1$.)

4. Let $k \in\{0,1\}$ such that

$\varphi_{i}(z) \neq\left\langle p\left(\right.\right.$ cur_index $\left._{s}\right), p($ cur_maxindex $)$, cur_notation $\left._{s}, k\right\rangle$. For $x_{s} \leq x \leq y$, let $\varphi_{p(0)}(x)=$ $\left\langle p\left(\right.\right.$ cur_index $\left._{s}\right), p($ cur_maxindex $s)$, cur_notation $\left._{s}, k\right\rangle$.

Change to next unused index: cur_index ${ }_{s+1}=$ cur_index $_{s}+1$.

Record diagonalized program: Cancel $_{s+1}=\operatorname{Cancel}_{s} \cup\{i\}$.

Carry forward unchanged all other global variables (cur_notation, cur_bound, cur_maxindex, and last_mindchange). 
Go to stage $s+1$. (Therefore $x_{s+1}=y+1$.)

5. For $x_{s} \leq x \leq y$, let $\varphi_{p(0)}(x)=\varphi_{p\left(\text { cur_index }_{s}\right)}(x)$.

Carry forward unchanged all global variables (cur_notation, cur_bound, cur_maxindex, last_mindchange, cur_index, and Cancel).

Go to stage $s+1$. (Therefore $x_{s+1}=y+1$.)

End stage $s$.

We now consider the following cases.

Case 1: Each stage is entered and terminates.

Since ordinals are well ordered, step 2.2 can succeed only finitely often. Thus, (as $s$ goes to infinity) cur_notation ${ }_{s}$, cur_bound ${ }_{s}$, cur_maxindex , last_mindchange $_{s}$ all stabilize to, say, cur_notation, cur_bound, cur_maxindex, last_mindchange. Now after the last success of step 2.2, step 2.3 can succeed only finitely often ( $\leq$ cur_bound +1 times, since each success of step 2.3 cancels a new program $\leq$ cur_bound). Thus for all but finitely many stages, step 2.4 must succeed. It follows, from steps 1 and 5 , that $\varphi_{p(\text { cur_index })}=\varphi_{p(0)}$ and $\mathbf{M}\left(\varphi_{p(\text { cur_index })}\right)$ outputs a program $>h(p(0), \infty)=g(p(0))$ infinitely often.

Clearly, $\varphi_{p(0)} \in \mathcal{C}_{u}$. Also, since $g$ is monotonically non-decreasing, $g(p(0)) \geq$ $g\left(\operatorname{MinProg}\left(\varphi_{p(0)}\right)\right)=g\left(\operatorname{MinProg}\left(\varphi_{p(\text { cur_index })}\right)\right)$. Therefore, either $\mathbf{M}\left(\varphi_{p(0)}\right) \uparrow$ or $\mathbf{M}\left(\varphi_{p(0)}\right) \downarrow \not L$ $g\left(\operatorname{MinProg}\left(\varphi_{p(0)}\right)\right)$.

Thus $\varphi_{p(0)} \in \mathcal{C}_{u}-g-\operatorname{Mex}(\mathbf{M})$.

Case 2: Stage $s$ is entered but does not terminate.

In this case $\varphi_{p\left(\text { cur_index }_{s}\right)} \in \mathcal{C}_{u}$, but $\mathbf{M}\left(\varphi_{p\left(\text { cur_index }_{s}\right)}\right)$ either does not converge, or converges to a program $\leq$ cur_bound $_{s}$. However each of the programs $\leq$ cur_bound $_{s}$ either convergently differs from $\varphi_{p \text { (cur_index }}$ ) (i.e., is in Cancel $_{s}$ ) or is undefined on all $x>x_{s}$ (since step 2.3 does not succeed). Thus, $\mathbf{M}$ does not $g$-Mex-identify $\varphi_{p \text { (cur_index }}$ ) $($ In fact, in this case, $\mathbf{M}$ does not even Ex-identify $\varphi\left(\operatorname{cur} \_\right.$index $\left.\left._{s}\right)\right)$.

Theorem 32 (General Hierarchy Theorem) For all $u \in O$, for all $w$ for $\omega, \mathbf{L i m}_{u} \mathbf{M e x} \subset$ $\operatorname{Lim}_{\left\{(u+o \underline{2})^{w}\right\}_{o}}$ Mex.

Proof. By Theorem 14(c), we get that for all $u, u<_{o}\left(u+_{o} \underline{2}\right)$, which, by Theorem 18(c), is $<_{o}\left\{\left(u+_{o} \underline{2}\right)^{w}\right\}_{o}$ (we recall that $\underline{2}=2^{2^{1}}=4>_{o} 2$ ). Hence, the theorem follows from Proposition 19 and Lemmas 31 and 30 .

Theorem 32 implies that there are infinite, infinitely ramifying hierarchies of parsimony restricted learning criteria. Furthermore, we have

Corollary 33 There are infinite hierarchies of parsimony restricted learning criteria which lie along $<_{o}$-paths, which paths have notations for each constructive ordinal.

Proof. First fix $w \in O$ for $\omega$. Let $O=\left\{x_{0}, x_{1}, x_{2}, \ldots\right\}$. Let $y_{0}=z_{0}=x_{0}$. Let $y_{n+1}=$ $\left\{\left(z_{n}+_{o} \underline{2}\right)^{w}\right\}_{o}$. Let $z_{n+1}=y_{n+1}+_{o} x_{n+1}$. Then, by Theorem 14 parts (c) and (e), for all $n$, $y_{n}<_{o}\left(y_{n}+_{o} x_{n}\right)+_{o} \underline{2}$. This latter, by Theorem 18(c), is $<_{o}\left\{\left(\left(y_{n}+_{o} x_{n}\right)+_{o} \underline{2}\right)^{w}\right\}_{o}$, which $=y_{n+1}$. Hence, the downward closure under $<_{o}$ of the set of $O$-notations, $\left\{y_{0}<_{o} y_{1}<_{o} y_{2}<_{o} \cdots\right\}$, is, for terminology from [Rog67], a maximal, univalent system of notations, and, from Proposition 19 and Theorem 32, $(\forall n \in N)\left[\mathbf{L i m}_{y_{n}} \mathbf{M e x} \subset \mathbf{L i m}_{y_{n+1}} \mathbf{M e x}\right]$.

As another Corollary to Theorem 32 and Proposition 19, we get 
Corollary 34 For all $u \in O, \operatorname{Lim}_{u}$ Mex $\subset$ LimMex.

Since $\operatorname{Lim}_{\underline{0}} \mathbf{M e x}=$ Mex, we get

\section{Corollary 35 Mex $\subset$ LimMex.}

Our next few results (Theorem 36, Lemma 37 and Lemma 39) lead up to another of our main results, our Strong Hierarchy Theorem (Theorem 40), which essentially states that for notations of the form $\left\{w^{v}\right\}_{o}$, where $v \in O$ and $w$ is for $\omega$, Theorem 20 gives as much collapsing as possible.

The following Theorem is a notational analog of Cantor's Normal Form (CNF) Theorem ${ }^{25}$ for ordinals ([Sie65, Theorem 2, Chapter XIV.19, page 323] and [KM67, Theorems 2 and 5, Chapter VII, Section 7]). ${ }^{26}$ In Theorem 36 below, as stated, the parenthesization of the $+_{o}$ terms to the right is essential, since $+_{o}$ is not associative.

Theorem 36 (Notational CNF Theorem) For all $v \in O$, for all $w$ for $\omega$, for all $x>_{o} \underline{0}$,

$$
x<_{o}\left\{w^{v}\right\}_{o} \Leftrightarrow\left\{\begin{array}{l}
\text { there exists a unique } k \in N \text {, unique } n_{0}, n_{1}, \ldots, n_{k} \in N^{+} \\
\text {and unique } v_{0}, v_{1}, \ldots, v_{k} \text { where } v>_{o} v_{0}>v_{1} \ldots>_{o} v_{k}, \text { such that } \\
x=\left\{w^{v_{0}}\right\}_{o} \times_{o} \underline{n_{0}}+_{o}\left(\left\{w^{v_{1}}\right\}_{o} \times_{o} \underline{n_{1}}+_{o}\left(\ldots+_{o}\left(\left\{w^{v_{k}}\right\}_{o} \times_{o} \underline{n_{k}}\right) \ldots\right)\right) .
\end{array}\right.
$$

Furthermore, for the left to right direction of the $\Leftrightarrow$ statement just above, the values of $k, n_{0}, \ldots, n_{k}$, and $v_{0}, \ldots, v_{k}$ can be algorithmically obtained from $v, w$, and $x$.

We call the above unique representation of $x<_{o}\left\{w^{v}\right\}_{o}$, for $v, w$, and $x$ as in the above theorem, the notational CNF of $x$ with respect to $\left\{w^{v}\right\}_{o}$. Where it is clear from the context, we will drop the phrase "with respect to $\left\{w^{v}\right\}_{o}$ " while referring to the notational CNF of such an $x$. Also, Theorem 36 above has several other variants that are also true; for example, we could get similar theorems for notations of the form $w_{1} \times_{o} w_{2} \times_{o} w_{3}$, or $\left\{w_{1}^{w_{2}}\right\}_{o} \times_{o} w_{3}$, where $w_{1}, w_{2}$ and $w_{3}$ are notations for $\omega$. We omit the details.

Furthermore, we note that for every ordinal $\alpha>0$, there exists a (unique) ordinal $\beta$ such that $\omega^{\beta} \leq \alpha<\omega^{\beta+1}$ [Sie65, Theorem 2, Chapter XIV.18, page 321]. Therefore, the above Notational CNF Theorem is sufficiently general to apply to notations for as large a constructive ordinal as we may choose.

\section{Proof of Notational CNF.}

We first prove the ' $\Leftrightarrow$ ' part of the theorem.

$(\Leftarrow)$ This direction of the proof uses reasoning and facts very similar to those used in the proofs of Claims 26 and 27 of Lemma 25. It uses the fact that, for all $w$ for $\omega$, and for all $n \in N$, for all $v^{\prime}, v^{\prime \prime} \in O, v^{\prime}<_{o} v^{\prime \prime} \Rightarrow\left\{w^{v^{\prime}}\right\}_{o} \times_{o} \underline{n}<_{o}\left\{w^{v^{\prime \prime}}\right\}_{o}$.

$(\Rightarrow)$ The proof is by transfinite induction on $v$. Suppose $v, w \in O$ such that $w$ is for $\omega$. Suppose $x \in O$ such that $\underline{0}<_{o} x<_{o}\left\{w^{v}\right\}_{o}$.

Base Case: $v=\underline{0}$. Since $\left\{w^{\underline{0}}\right\}_{o}=\underline{1}$, this case is vacuously true.

Inductive Case: $v>_{o} \underline{0}$.

Inductive Hypothesis $(I H)$ : The $(\Rightarrow)$ direction holds for all notations $<_{o} v$.

\footnotetext{
${ }^{25}$ The CNF Theorem states: for any ordinal $\beta>0$, there exists a unique $k$, unique $n_{0}, n_{1}, \ldots, n_{k} \in N^{+}$, and unique ordinals $\alpha_{0}, \alpha_{1}, \ldots, \alpha_{k}$, where $\alpha_{0}>\alpha_{1}>\ldots>\alpha_{k}$, such that $\beta=\omega^{\alpha_{0}} \times n_{0}+\omega^{\alpha_{1}} \times n_{1}+\ldots+\omega^{\alpha_{k}} \times n_{k}$.

${ }^{26}$ For a web page describing a programmatic implementation of an algorithm that may be used to perform operations such as addition, subtraction, etc., on ordinals represented in Cantor Normal Form, see [Beh97].
} 
We will show that the $(\Rightarrow)$ direction holds for $v$.

Subcase 1: $v=u+o \underline{1}$.

Clearly, either $x<_{o}\left\{w^{u}\right\}_{o}$ or $\left\{w^{u}\right\}_{o} \leq_{o} x<_{o}\left\{w^{u+{ }_{o} \underline{1}}\right\}_{o}$. If the former is true, the $(\Rightarrow)$ direction follows from IH. Therefore, suppose the latter. Then, by Theorem 16(f), there exists a unique $n \in N^{+}$and a unique $x^{\prime}<_{o}\left\{w^{u}\right\}_{o}$ such that $x=\left\{w^{u}\right\}_{o} \times_{o} \underline{n}+_{o} x^{\prime}$. If $x^{\prime}=\underline{0}$, then clearly the $(\Rightarrow)$ direction holds with $k=0, v_{0}=u$, and $n_{0}=n$; otherwise, if $x>_{o} \underline{0}$, the $(\Rightarrow)$ direction follows by applying IH to $x^{\prime}$ and noting that $v>_{o} u$.

Subcase $2: v=3 \cdot 5^{p}$.

Let $\left\{w^{v}\right\}_{o}=3 \cdot 5^{q}$. Therefore, by Fact $7(\mathrm{e}),(\exists n \in N)\left[x<_{o} \varphi_{q}(n)\right]$. From Theorem 17,

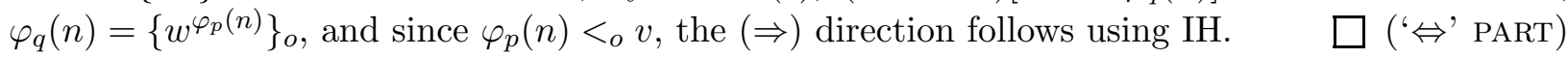

Finally, we prove the 'furthermore' part of the theorem. Suppose $v, w \in O$ such that $w$ is for $\omega$. Suppose $x \in O$ such that $\underline{0}<_{o} x<_{o}\left\{w^{v}\right\}_{o}$. By using Fact 8(a) we can enumerate, uniformly effectively in $v$ and $w$, the set $S=\left\{\left(k, n_{0}, n_{1}, \ldots, n_{k}, v_{0}, v_{1}, \ldots, v_{k}\right) \mid k \in\right.$ $N, n_{0}, n_{1}, \ldots, n_{k} \in N^{+}, v>_{o} v_{0}>_{o} v_{1} \ldots>_{o} v_{k}$ and $\left\{w^{v_{0}}\right\}_{o} \times_{o} n_{0}+_{o}\left(\left\{w^{v_{1}}\right\}_{o} \times_{o} n_{1}+_{o}\left(\ldots+_{o}\right.\right.$ $\left.\left.\left.\left(\left\{w^{v_{k}}\right\}_{o} \times_{o} n_{k}\right) \ldots\right)\right)<_{o}\left\{w^{v}\right\}_{o}\right\}$. Clearly, then, there is a procedure that is effective in $v, w$, and $x$ to find $\left(k, n_{0}, n_{1}, \ldots, n_{k}, v_{0}, v_{1}, \ldots, v_{k}\right) \in S$ such that $x=\left\{w^{v_{0}}\right\}_{o} \times_{o} \underline{n_{0}}+_{o}\left(\left\{w^{v_{1}}\right\}_{o} \times_{o} \underline{n_{1}}+_{o}(\ldots+o\right.$ $\left.\left.\left(\left\{w^{v_{k}}\right\}_{o} \times_{o} \underline{n_{k}}\right) \ldots\right)\right)$.

(Notational CNF Theorem)

We next present a lemma (Lemma 37) which is a strengthening of Lemma 25 for notations in certain forms. In Lemma 25, if we let $u=\left\{w^{v}\right\}_{o}$, for some notation $v \in O$ and some $w$ for $O$, then we get that every $\lim _{u}$-computable function is dominated by a parsimony factor of order $\left\{\left(\left\{w^{v}\right\}_{o}+_{o} \underline{1}\right)^{w}\right\}_{o}$. Lemma 37, however, implies that we can in fact do much better and use parsimony factors of order just $\left\{w^{v+o} \underline{1}\right\}_{o}$.

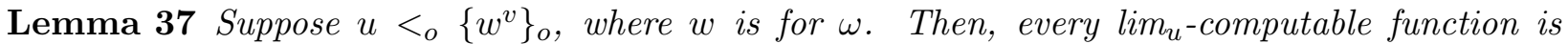
dominated by a monotonically non-decreasing $\lim _{\left\{w^{v}\right\}_{o}}$-computable function.

\section{Proof of Lemma 37.}

Suppose $v, w \in O$ such that $w$ is for $\omega$. If $u=\underline{0}$, then the lemma follows using reasoning similar to that in the proof of Lemma 25. For the $u>_{o} \underline{0}$ cases, we will use Lemma 24 .

Firstly, for all notations $x, y<_{o}\left\{w^{v}\right\}_{o}$, we define the natural sum of $x$ and $y$ and denote the operation by $(+)_{o}$; this operation is a notational analog of the natural sum of ordinals from [KM67, Chapter VII, Section 7, pages 259-260]. ${ }^{27}$

Suppose $x, y>_{o} \underline{0}$. Let $x$ 's and $y$ 's (unique) notational CNFs, obtained via the Notational CNF Theorem 36, be

$$
\begin{aligned}
& x=\left\{w^{v_{0}^{\prime}}\right\}_{o} \times_{o} \underline{n_{0}^{\prime}}+_{o}\left(\left\{w^{v_{1}^{\prime}}\right\}_{o} \times_{o} \underline{n_{1}^{\prime}}+_{o}\left(\ldots+_{o}\left(\left\{w^{v_{k^{\prime}}^{\prime}}\right\}_{o} \times_{o} \underline{n_{k^{\prime}}^{\prime}}\right) \ldots\right)\right), \\
& y=\left\{w^{v_{0}^{\prime \prime}}\right\}_{o} \times_{o} \underline{n_{0}^{\prime \prime}}+_{o}\left(\left\{w^{v_{1}^{\prime \prime}}\right\}_{o} \times_{o} \underline{n_{1}^{\prime \prime}}+_{o}\left(\ldots++_{o}\left(\left\{w^{v_{k^{\prime \prime}}^{\prime \prime}}\right\}_{o} \times_{o} \underline{n_{k^{\prime \prime}}^{\prime \prime}}\right) \ldots\right)\right),
\end{aligned}
$$

where $k^{\prime}, k^{\prime \prime} \in N, n_{0}^{\prime}, \ldots, n_{k^{\prime}}^{\prime}, n_{0}^{\prime \prime}, \ldots, n_{k^{\prime \prime}}^{\prime \prime} \in N^{+}, v>_{o} v_{0}^{\prime} \ldots>_{o} v_{k^{\prime}}^{\prime}$, and $v>_{o} v_{0}^{\prime \prime} \ldots>_{o} v_{k^{\prime \prime}}^{\prime \prime}$. (We note that in general, $k^{\prime}$ may be different from $k^{\prime \prime}$.)

By Fact $7(\mathrm{f})$, we get that for all $v^{\prime} \in V^{\prime}=\left\{v_{i}^{\prime} \mid i \leq k^{\prime}\right\}$, for all $v^{\prime \prime} \in V^{\prime \prime}=\left\{v_{i}^{\prime \prime} \mid i \leq k^{\prime \prime}\right\}$, either $v^{\prime}<_{o} v^{\prime \prime}$ or $v^{\prime}=_{o} v^{\prime \prime}$ or $v^{\prime}>_{o} v^{\prime \prime}$. Let $v_{0}>_{o} v_{1} \ldots>_{o} v_{k}$ be the notations in $V^{\prime} \cup V^{\prime \prime}$.

\footnotetext{
${ }^{27}$ N.B. our notational $(+)_{o}$ depends on the choice of the pre-given $v$ and $w$.
} 
By Theorem 15 (clause i), we have that for all $x, x \times_{o} \underline{0}=\underline{0}$. Also, as we noted a few paragraphs after Theorem 13, clause (i) of Theorem 13 is a special base case that we added to Kleene's definition of $+_{o}$ from [Kle55], in order to get that for all $y, \underline{0}+_{o} y=y$. Thus, we have that for any $x, y, \in O$, $\left(x \times_{o} \underline{0}\right)+{ }_{o} y=y$.

Using the facts from the just above paragraph, then, there is exactly one way to write $x$ and $y$ as

$$
\begin{aligned}
& x=\left\{w^{v_{0}}\right\}_{o} \times_{o} \underline{m_{0}^{\prime}}+_{o}\left(\left\{w^{v_{1}}\right\}_{o} \times_{o} \underline{m_{1}^{\prime}}+_{o}\left(\ldots+_{o}\left(\left\{w^{v_{k}}\right\}_{o} \times_{o} \underline{m_{k}^{\prime}}\right) \ldots\right)\right), \\
& y=\left\{w^{v_{0}}\right\}_{o} \times_{o} \underline{m_{0}^{\prime \prime}}+_{o}\left(\left\{w^{v_{1}}\right\}_{o} \times_{o} \underline{m_{1}^{\prime \prime}}+_{o}\left(\ldots+{ }_{o}\left(\left\{w^{v_{k}}\right\}_{o} \times_{o} \underline{m_{k}^{\prime \prime}}\right) \ldots\right)\right),
\end{aligned}
$$

where $m_{0}^{\prime}, \ldots, m_{k}^{\prime}$ and $m_{0}^{\prime \prime}, \ldots, m_{k}^{\prime \prime}$ are possibly 0 , and $v>_{o} v_{0}$. Furthermore, we clearly can still effectively determine all notations involved in the above representations of $x$ and $y$. We call the immediately above forms of $x$ and $y$ the mutually completed notational CNFs of $x$ and $y$. It is similarly possible to extend the definition of mutual completion of notational CNFs to sequences of more than two notations. We omit the details.

Now, the natural sum of $x$ and $y$ : If $x=\underline{0}$ or $y=\underline{0}$,

$$
x(+)_{o} y \stackrel{\text { def }}{=} x+o y
$$

Otherwise, $x(+)_{o} y \stackrel{\text { def }}{=}$

$$
\left\{w^{v_{0}}\right\}_{o} \times_{o} \underline{\left(m_{0}^{\prime}+m_{0}^{\prime \prime}\right)}+_{o}\left(\left\{w^{v_{1}}\right\}_{o} \times_{o} \underline{\left(m_{1}^{\prime}+m_{1}^{\prime \prime}\right)}+_{o}\left(\ldots+_{o}\left(\left\{w^{v_{k}}\right\}_{o} \times_{o} \underline{\left(m_{k}^{\prime}+m_{k}^{\prime \prime}\right)}\right) \ldots\right)\right) .
$$

Clearly, $(+)_{o}$ is an effective operation. It is also commutative and associative, unlike $+_{o}$. Next, we prove the following properties of $(+)_{0}$.

Claim 38 Suppose $v, x, y, w \in O$ such that $w$ is for $\omega$. Suppose $x, y<_{o}\left\{w^{v}\right\}_{o}$. Then,

(a) $x(+)_{o} y<_{o}\left\{w^{v}\right\}_{o}$

(b) $\left(\forall y^{\prime}\right)\left[y^{\prime}<_{o} y \Rightarrow\left(x(+)_{o} y^{\prime}<_{o} x(+)_{o} y\right)\right]$,

(c) $\left(\forall x^{\prime}\right)\left[x^{\prime}<_{o} x \Rightarrow\left(x^{\prime}(+)_{o} y<_{o} x(+)_{o} y\right)\right]$.

\section{Proof of Claim 38.}

The proof is fairly straightforward, and, hence, we only sketch the details. Part (a) may be proved along the lines of Claim 27 of Lemma 25. Part (c) follows from Part (b) and the commutativity of $(+)_{o}$.

Part (b) may be proved by transfinite induction. In the induction, we use the fact that if the leftmost term of (the notational CNF of) $y$ that is different from the corresponding term of (the notational CNF of $) y^{\prime}$ is $\left\{w^{v_{0}}\right\}_{o} \times_{o} \underline{n_{0}}$ and the corresponding term of $y^{\prime}$ is $\left\{w^{v_{0}^{\prime}}\right\}_{o} \times_{o} \underline{n_{0}^{\prime}}$, then either $v_{0}^{\prime}<_{o} v_{0}$ or $\left(v_{0}^{\prime}=v_{0} \wedge n_{0}^{\prime}<n_{0}\right)$. Then, we consider the mutually completed notational CNFs of $x(+)_{o} y$ and $x(+)_{o} y^{\prime}$ and use a proof that is similar to that of the proofs of Claims 26 and 27 of Lemma 25, to show that $x(+)_{o} y^{\prime}<_{o} x(+)_{o} y$.

(Claim 38)

Next, we define a family of partial computable functions $\left\{\psi_{n} \mid n \in N\right\}$ that will enable us to use Lemma 24 to get the desired result. For all $n$, for all $u_{0}, \ldots, u_{n} \leq_{o} u<_{o}\left\{w^{v}\right\}_{o}$, let

$$
\psi_{n}\left(u_{n}, \ldots, u_{0}\right)=u_{0}(+)_{o} \ldots(+)_{o} u_{n} .
$$

Clearly, using Claim 38, the definition of $(+)_{o}$ and the fact that $u>_{o} \underline{0}$, we see that $u$ and $\left\{\psi_{n} \mid n \in N\right\}$ satisfy the hypotheses of Lemma 24. Also, using Claim 38, it is easy to show 
that $\alpha=\lim _{n \rightarrow \infty}\left|\psi_{n}(u, \ldots, u)\right|_{o} \leq\left|\left\{w^{v}\right\}_{o}\right|_{o}$. Let $v^{*}$ be the unique notation for $\alpha$ such that $v^{*} \leq_{o}\left\{w^{v}\right\}_{o}$. Therefore, we have $u<_{o} v^{*} \leq_{o}\left\{w^{v}\right\}_{o}$.

Lastly, applying Lemma 24 to $u,\left\{\psi_{n} \mid n \in N\right\}$ and $v^{*}$, Lemma 37 follows.

(LEMMA 37)

As an application of Lemma 37, we get the following. It is important to note that, for any notations $v, w$, where $w$ is for $\omega$, the notations $\left(\left\{w^{v}\right\}_{o} \times_{o} w\right)$ and $\left\{w^{v+{ }_{o}} \underline{1}\right\}_{o}$ are not only for the same ordinal, but also are exactly the same number, i.e., notation (this follows from our carefully chosen "definitions" of $\times_{o}$ and $\left.\{\cdot\}_{o}\right)$.

Lemma 39 For all $v, w \in O$, where $w$ is for $\omega, \mathcal{C}_{\left\{w^{v}\right\}_{o}} \in \operatorname{Lim}_{\left\{w^{v}\right\}_{o} \times_{o} w}$ Mex.

Proof. Follows from Lemmas 29 and 37, using reasoning similar to that presented just before Lemma 30.

As indicated earlier, we get, for notations in special form, the following strengthening of the General Hierarchy Theorem (Theorem 32).

Theorem 40 (Strong Hierarchy Theorem) Suppose $u, v, w \in O$ where $w$ is for $\omega$. Then, (a) $\left[\left\{w^{v}\right\}_{o} \leq_{o} u<_{o}\left\{w^{v}\right\}_{o} \times_{o} w\right] \Rightarrow \operatorname{Lim}_{u} \mathbf{M e x}=\mathbf{L i m}_{\left\{w^{v}\right\}_{o}} \mathbf{M e x}$,

(b) $\operatorname{Lim}_{\left\{w^{v}\right\}_{o}} \operatorname{Mex} \subset \operatorname{Lim}_{\left\{w^{v}\right\}_{o} \times_{o} w} \operatorname{Mex}$,

(c) $v$ is a notation for a limit ordinal $\Rightarrow\left[\right.$ for all $\left.u<_{o} v, \operatorname{Lim}_{\left\{w^{u}\right\}_{o}} \mathbf{M e x} \subset \mathbf{L i m}_{\left\{w^{v}\right\}_{o}} \mathbf{M e x}\right]$.

Proof. (a) Follows from Theorem 20.

(b) Follows from Lemmas 31 and 39, and Proposition 19.

(c) Suppose $v \in O$ is for a limit ordinal and $v^{\prime}<_{o} v$. By part (b), we have $\operatorname{Lim}_{\left\{w^{v^{\prime}}\right\}_{o}} \mathbf{M e x} \subset$ $\operatorname{Lim}_{\left\{w^{\left.\left(v^{\prime}+o \underline{1}\right)\right\}_{o}}\right.}$ Mex. Also, since $v$ is for a limit ordinal, $\left(v^{\prime}+{ }_{o} \underline{1}\right)<_{o} v{ }^{28}$ Hence, by Theorem 18(d), $\left\{w^{\left(v^{\prime}+{ }_{o} \underline{1}\right)}\right\}_{o}<_{o}\left\{w^{v}\right\}_{o}$. Therefore, by Proposition 19, $\operatorname{Lim}_{\left\{w^{\left(v^{\prime}+o \underline{1}\right)}\right\}_{o}} \mathbf{M e x} \subseteq \operatorname{Lim}_{\left\{w^{v}\right\}_{o}}$ Mex. Thus $\operatorname{Lim}_{\left\{w^{v^{\prime}}\right\}_{o}} \operatorname{Mex} \subset \operatorname{Lim}_{\left\{w^{v}\right\}_{o}} \operatorname{Mex}$.

As a corollary to Theorem 40, we get

Corollary 41 Suppose $u, v, w \in O$, where $w$ is for $\omega$. Then, $u<_{o}\left\{w^{v}\right\}_{o} \Rightarrow \operatorname{Lim}_{u} \operatorname{Mex} \subset \operatorname{Lim}_{\left\{w^{v}\right\}_{o}}$ Mex.

ProOF.

Follows using an easy induction on $v$, along with Proposition 19, Theorems 36 and 40, and Fact $7(\mathrm{e})$.

Next, suppose $n \in N$ and $w$ is for $\omega$. Then, Theorem 40 gives us, for example, (i) $\operatorname{Lim}_{\{w \underline{n}\}_{o}} \operatorname{Mex} \subset \operatorname{Lim}_{\left\{w \frac{n+1}{\}_{o}}\right\}_{o}}$ Mex; (ii) $\operatorname{Lim}_{\{w \underline{n}\}_{o}} \operatorname{Mex} \subset \operatorname{Lim}_{\left\{w^{w}\right\}_{o}}$ Mex, and (iii)

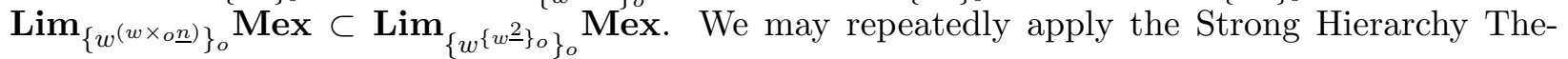
orem to get a hierarchy of the form shown in Figure 1, Section 1.3.

Ordinal $\epsilon_{0}$ is the limit of the sequence $\omega, \omega^{\omega}, \omega^{\omega^{\omega}}, \ldots$ as well as the least fixed point of $\lambda \beta\left[\omega^{\beta}\right]$. Furthermore, it is the least ordinal not expressible as a polynomial in $0,1, \ldots, \omega$ using finitely many applications of addition, multiplication and exponentiation. ${ }^{29}$ The example of Figure 1 may be extended to notations for $\epsilon$ numbers and beyond by a strategy we illustrate for $\epsilon_{0}$. Let

$$
S=\left\{u \mid u \leq_{o} \text { some notation in } w,\left\{w^{w}\right\}_{o},\left\{w^{\left\{w^{w}\right\}_{o}}\right\}_{o}, \ldots\right\}
$$

\footnotetext{
${ }^{28}$ Proof: Since $v^{\prime}<_{o} v$, by Fact $7(\mathrm{~d})$, we get $v^{\prime}+{ }_{o} \underline{1} \leq_{o} v$. Since $v$ is a limit ordinal, $v^{\prime}+_{o} \underline{1} \neq v$. Hence $v^{\prime}+_{o} \underline{1}<_{o} v$. ${ }^{29}$ See [Rog67, Exercises 11-51 to 11-53, Page 221].
} 
Let $e^{\prime}$ be such that for all $n$, (a) $\varphi_{e^{\prime}}(n) \in S$, (b) $\varphi_{e^{\prime}}(n)<_{o} \varphi_{e^{\prime}}(n+1)$, and (c) for all $u \in S$, there exists an $n$ such that $u \leq_{o} \varphi_{e^{\prime}}(n)$. Then, clearly, $3 \cdot 5^{e^{\prime}} \in O$ and $e=3 \cdot 5^{e^{\prime}}$ is a notation for $\epsilon_{0}$. Choosing any such $e$ for $\epsilon_{0}$, we can employ $\left\{w^{e}\right\}_{o}$ as another notation for $\epsilon_{0}$ and continue the hierarchy of Figure 1, by that shown in Figure 2 below, for example.

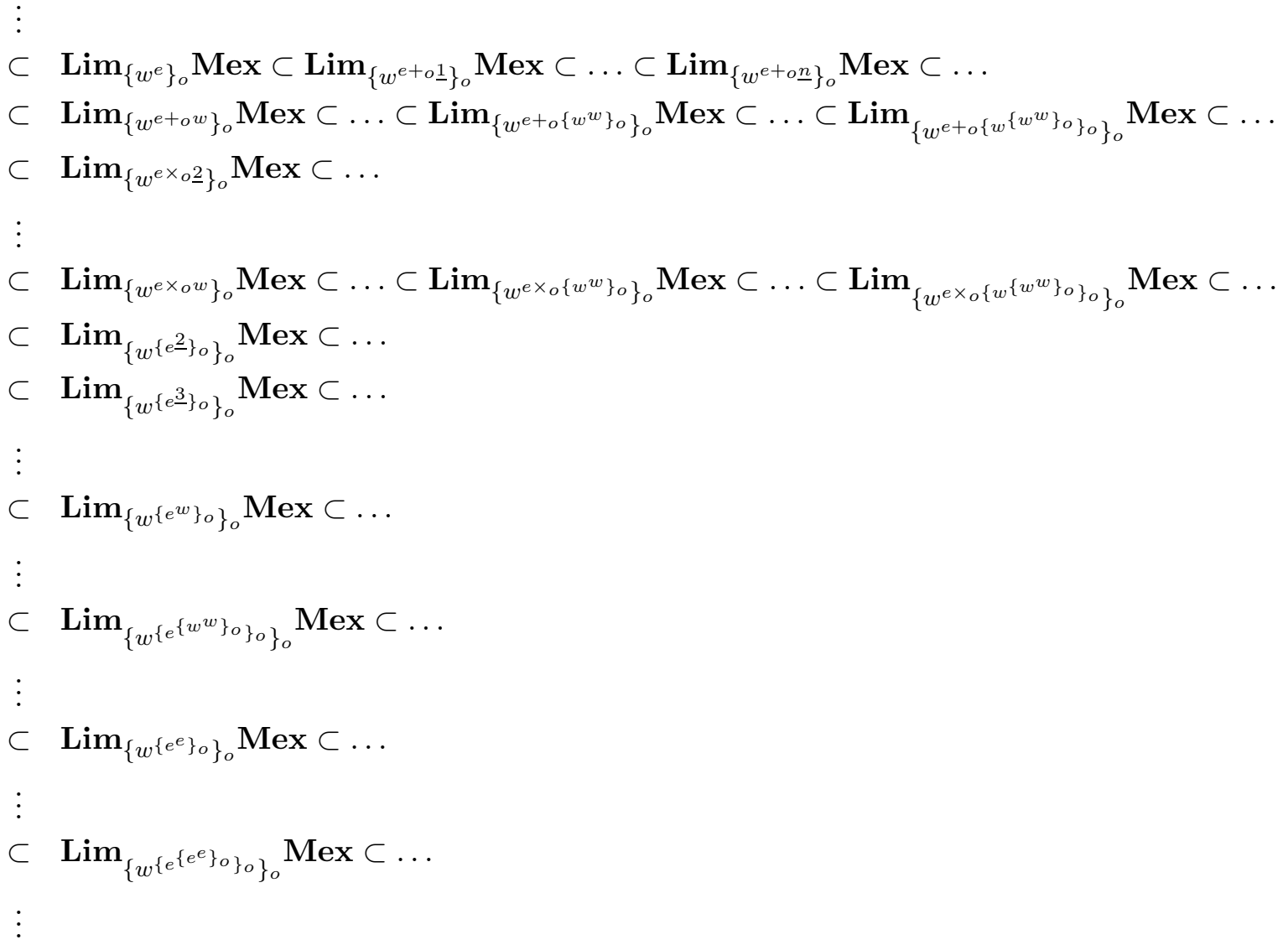

Figure 2: Example optimal continuation of hierarchy of Figure 1 along a $<_{o}$-path for ordinals $<\epsilon_{1}$.

We have not attempted to obtain notational analogs of ordinal normal form theorems other than for Cantor's Normal Form Theorem but expect there are such results which would yield interesting Strong Hierarchies for the $\mathbf{L i m}_{u} \mathbf{M e x}$ criteria and which are based on notations, e.g., for the $\epsilon$ ordinals, which do not have to be of the form $\left\{w^{v}\right\}_{o}$ (for example, $e$ just above).

\section{Further Results and Future Work}

As stated in Theorem 23, for all $n \in O, \mathbf{L i m}_{\underline{n}} \mathbf{M e x}=\mathbf{M e x}$. We had originally hoped that this result would not be true and that there would be a fine hierarchy between Mex and LimMex based on $\lim _{\underline{n}}$-computable parsimony factors. In the next section we successfully explore some different sources of restricted-parsimony fine structure. 


\subsection{Iterated-Limits Parsimony Factors}

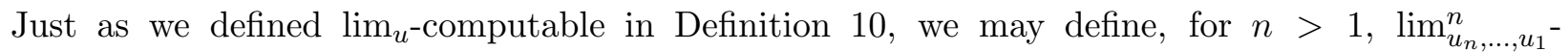
computable functions. These $\lim _{u_{n}, \ldots, u_{1}}^{n}$-computable functions are used in the definitions of corresponding $\mathbf{L i m}_{u_{n}, \ldots, u_{1}}^{n}$ Mex learning criteria.

We can extend Definition 4 to

Definition 42 Suppose $n>0$. Suppose $h: N^{n+1} \rightarrow N$. Then,

(a) for all $x$, and any sequence of $n-1$ numbers $t_{1}, \ldots, t_{n-1}$,

$$
\lim _{t \rightarrow \infty} h\left(x, t_{n-1}, \ldots, t_{1}, t\right) \stackrel{\text { def }}{=} \begin{cases}y, & \text { if }\left(\forall^{\infty} t\right)\left[h\left(x, t_{n-1}, \ldots, t_{1}, t\right)=y\right] ; \\ \uparrow, & \text { otherwise. }\end{cases}
$$

(b) for each $m$ such that $0<m \leq n$, for any sequence of $n-m$ numbers $t_{m}, \ldots, t_{n-1}$,

$$
h\left(x, t_{n-1}, \ldots, t_{m}, \infty, \ldots, \infty\right) \stackrel{\text { def }}{=} \lim _{t \rightarrow \infty} h\left(x, t_{n-1}, \ldots, t_{m}, t, \infty, \ldots, \infty\right) .
$$

Definition 43 Suppose $m, n \in N$ such that $0<m \leq n$. Then, $\mathbf{F}: N^{n+1} \rightarrow O$ is an $(m, n) O$-countdown function $\stackrel{\text { def }}{\Leftrightarrow}$ for all $x$, for all sequences of $n-m+1$ numbers $t_{m}, \ldots, t_{n}, \mathbf{F}\left(x, t_{n}, \ldots, t_{m}+1, \infty, \ldots, \infty\right) \leq_{o} \mathbf{F}\left(x, t_{n}, \ldots, t_{m}, \infty, \ldots, \infty\right)$.

We next illustrate our general definition for, $n>0$, of $\lim _{u_{n}, \ldots, u_{1}}^{n}$-computable functions, via the $n=2$ case.

Definition 44 Suppose $u_{2}, u_{1} \in(O \cup\{*\})$. Then, $g: N \rightarrow N$ is $\lim _{u_{2}, u_{1}}^{2}$-computable $\stackrel{\text { def }}{\Leftrightarrow}$ there exists computable $h$ such that, for all $x, t_{1}, t_{2}$

(a) $g(x)=h(x, \infty, \infty)$,

(b) $\operatorname{card}\left(\left\{t \mid h\left(x, t_{2}, t\right) \neq h\left(x, t_{2}, t+1\right)\right\}\right)$ is finite,

(c) $\operatorname{card}(\{t \mid h(x, t, \infty) \neq h(x, t+1, \infty)\})$ is finite,

(d) $\left(u_{1} \neq *\right) \Rightarrow$ there exists a $(1,2) O$-countdown function $\mathbf{F}_{1}$ such that

$\mathbf{F}_{1}\left(x, t_{2}, 0\right) \leq_{o} u_{1} \wedge$

and$$
h\left(x, t_{2}, t_{1}\right) \neq h\left(x, t_{2}, t_{1}+1\right) \Rightarrow \mathbf{F}_{1}\left(x, t_{2}, t_{1}+1\right)<_{o} \mathbf{F}_{1}\left(x, t_{2}, t_{1}\right),
$$

(e) $\left(u_{2} \neq *\right) \Rightarrow$ there exists a $(2,2) O$-countdown function $\mathbf{F}_{2}$ such that

$\mathbf{F}_{2}\left(x, t_{2}, \infty\right) \leq_{o} u_{2} \wedge$

$h\left(x, t_{2}, \infty\right) \neq h\left(x, t_{2}+1, \infty\right) \Rightarrow \mathbf{F}_{2}\left(x, t_{2}+1, \infty\right)<_{o} \mathbf{F}_{2}\left(x, t_{2}, \infty\right)$.

We define lim $_{u_{2}, u_{1}}^{2}$-computable as witnessed by $h, \mathbf{F}_{1}$, and $\mathbf{F}_{2}$ along the lines of part (b) of Definition 5; we omit the details.

Let $\underline{N} \stackrel{\text { def }}{=}\{\underline{n} \mid n \in N\}$. It turns out that $\lim _{u_{n}, \ldots, u_{1}}^{n}$-computable functions, where each $u_{i} \in(\underline{N} \cup$ $\{*\})$, have useful characterizations as stated in Proposition 45 below. When we are proving results for the $\mathbf{L i m}_{u_{n}, \ldots, u_{1}}^{n}$ Mex criteria where each $u_{i} \in(\underline{N} \cup\{*\})$, we shall use these characterizations in our proofs involving iterated limits below, since, then, we do not have to explicitly consider the more complicated corresponding $(m, n) O$-countdown functions.

Proposition 45 Suppose $n>0$ and $u_{1}, \ldots, u_{n} \in(\underline{N} \cup\{*\})$. Then, $g: N \rightarrow N$ is $\lim _{u_{n}, \ldots, u_{1}}^{n}{ }^{-}$ computable $\Leftrightarrow$ there exists a computable $h: N^{n+1} \rightarrow N$ such that for all $x$,

(a) $g(x)=h(x, \infty, \ldots, \infty)$,

(b) for all $i$ such that $1 \leq i<n$ and $u_{i} \neq *$, for any sequence of $n-i$ numbers $t_{n}, \ldots, t_{i+1}$, $\operatorname{card}\left(\left\{t \mid h\left(x, t_{n}, \ldots, t_{i+1}, t, \infty, \ldots, \infty\right) \neq h\left(x, t_{n}, \ldots, t_{i+1}, t+1, \infty, \ldots, \infty\right)\right\}\right) \leq\left|u_{i}\right|_{o}$, and

(c) for all $i$ such that $1 \leq i<n$ and $u_{i}=*$, for any sequence of $n-i$ numbers $t_{n}, \ldots, t_{i+1}$, $\operatorname{card}\left(\left\{t \mid h\left(x, t_{n}, \ldots, t_{i+1}, t, \infty, \ldots, \infty\right) \neq h\left(x, t_{n}, \ldots, t_{i+1}, t+1, \infty, \ldots, \infty\right)\right\}\right)$ is finite. 
We write $\lim ^{n}$-computable for $\lim _{*, \ldots, *}^{n}$-computable. For $n>0$, we define $\mathbf{L i m}_{u_{n}, \ldots, u_{1}}^{n} \mathbf{M e x}$ just as in Definition 11, except that we use $\lim _{u_{n}, \ldots, u_{1}}^{n}$-computable parsimony factors instead of $\lim _{u^{-}}$ computable ones. We mostly write $\mathbf{L i m}^{n} \mathbf{M e x}$ instead of $\mathbf{L i m}_{*, \ldots, *}^{n} \mathbf{M e x}$.

For parsimony factors computed by two levels of iterated limits, the resulting learning criteria turn out to be, in some cases, identical to Mex, and in other cases, to LimMex. This is illustrated by the next two results. Proposition 46 just below is easy to prove.

\section{Proposition 46}

(a) For $u \in(O \cup\{*\}), \mathbf{L i m}_{u, \underline{0}}^{2} \mathbf{M e x}=\mathbf{L i m}_{\underline{0}, u}^{2} \mathbf{M e x}=\mathbf{L i m}_{u} \mathbf{M e x}$.

(b) For $u_{1}, u_{2}, v_{1}, v_{2} \in O, u_{1} \leq_{o} v_{1} \wedge u_{2} \leq_{o} v_{2} \Rightarrow \mathbf{L i m}_{\underline{0}, \underline{0}}^{2} \mathbf{M e x} \subseteq \mathbf{L i m}_{u_{2}, u_{1}}^{2} \mathbf{M e x} \subseteq$ $\mathbf{L i m}_{v_{2}, v_{1}}^{2} \mathbf{M e x} \subseteq \mathbf{L i m}^{2} \mathbf{M e x}$.

Theorem 47 For any notations $w_{1}$ and $w_{2}$ for $\omega, \mathbf{L i m}_{w_{2}, w_{1}}^{2} \mathbf{M e x}=\mathbf{E x}$.

Proof. $(\subseteq)$ Follows from the definition of $\mathbf{L i m}_{w_{2}, w_{1}}^{2}$ Mex.

$(\supseteq)$ Suppose $\mathcal{S} \in \mathbf{E x}$ as witnessed by $\mathbf{M}$.

We first define the following two (computable) predicates. For each $i, t_{1}$ and $t_{2}$, let $P\left(i, t_{2}, t_{1}\right) \equiv$ $\left(\forall x<t_{2}\right)\left[\Phi_{i}(x) \leq t_{1}\right]$ and $Q\left(i, t_{2}, t_{1}\right) \equiv\left(\forall y \mid t_{2} \leq y \leq t_{1}\right)\left[(\forall x<y)\left[\Phi_{i}(x) \leq t_{1}\right] \Rightarrow\left[\mathbf{M}\left(\varphi_{i}\left[t_{2}\right]\right)=\right.\right.$ $\left.\left.\mathbf{M}\left(\varphi_{i}[y]\right)\right]\right]$.

Also, for all $x, t_{2}$, let $P\left(i, t_{2}, \infty\right)=\lim _{t \rightarrow \infty} P\left(i, t_{2}, t\right)$ and $Q\left(i, t_{2}, \infty\right)=\lim _{t \rightarrow \infty} Q\left(i, t_{2}, t\right)$; then $P\left(i, t_{2}, \infty\right) \equiv\left(\forall x<t_{2}\right)\left[\varphi_{i}(x) \downarrow\right]$ and $Q\left(i, t_{2}, \infty\right) \equiv\left(\forall y \geq t_{2}\right)\left[(\forall x<y)\left[\varphi_{i}(x) \downarrow\right] \Rightarrow \mathbf{M}\left(\varphi_{i}\left[t_{2}\right]\right)=\right.$ $\left.\mathbf{M}\left(\varphi_{i}[y]\right)\right]$.

We next define $h^{\prime}$ as follows. It is to be understood, that for values of $h^{\prime}$, any ?'s are changed to 0's.

$$
h^{\prime}\left(i, t_{2}, t_{1}\right)= \begin{cases}\mathbf{M}\left(\varphi_{i}\left[t_{2}\right]\right), & \text { if } P\left(i, t_{2}, t_{1}\right) \wedge Q\left(i, t_{2}, t_{1}\right) \\ 0, & \text { otherwise }\end{cases}
$$

The following can be easily verified.

(1) $(\forall i)\left(\forall t_{1}, t_{2}\right)\left[P\left(i, t_{2}, t_{1}\right) \Rightarrow P\left(i, t_{2}, t_{1}+1\right)\right]$.

(2) $(\forall i)\left(\forall t_{1}, t_{2}\right)\left[\neg Q\left(i, t_{2}, t_{1}\right) \Rightarrow \neg Q\left(i, t_{2}, t_{1}+1\right)\right]$.

(3) $(\forall i)\left(\forall t_{1}, t_{2}\right)\left[\neg P\left(i, t_{2}, \infty\right) \Rightarrow \neg P\left(i, t_{2}+1, \infty\right)\right]$.

(4) $(\forall i)\left(\forall t_{1}, t_{2}\right)\left[Q\left(i, t_{2}, \infty\right) \Rightarrow Q\left(i, t_{2}+1, \infty\right)\right]$.

Item (1) implies that for all $i, t_{2}, \operatorname{card}\left(\left\{t \mid P\left(i, t_{2}, t\right) \neq P\left(i, t_{2}, t+1\right)\right\}\right) \leq 1$. Item (2) implies that for all $i, t_{2}, \operatorname{card}\left(\left\{t \mid Q\left(i, t_{2}, t\right) \neq Q\left(i, t_{2}, t+1\right)\right\}\right) \leq 1$. Hence, for all $i, t_{2}, \operatorname{card}\left(\left\{t \mid\left(P\left(i, t_{2}, t\right) \wedge\right.\right.\right.$ $\left.\left.\left.Q\left(i, t_{2}, t\right)\right) \neq\left(P\left(i, t_{2}, t+1\right) \wedge Q\left(i, t_{2}, t+1\right)\right)\right\}\right) \leq 2$. Therefore, from the definition of $h^{\prime}$, we get that for all $i, t_{2}, \operatorname{card}\left(\left\{t \mid h^{\prime}\left(i, t_{2}, t\right) \neq h^{\prime}\left(i, t_{2}, t+1\right)\right\}\right) \leq 2$. Similarly, from items (3) and (4), we can conclude that $\operatorname{card}\left(\left\{t \mid h^{\prime}(i, t, \infty) \neq h^{\prime}(i, t+1, \infty)\right\}\right) \leq 2$.

Let $g^{\prime}(i)=h^{\prime}(i, \infty, \infty)$. Hence, $g^{\prime}$ is a not necessarily monotonically non-decreasing, $\lim _{2,2^{-}}^{2}$ computable function. It is easy to verify that, for all $i$, if $\varphi_{i} \in \mathbf{E x}(\mathbf{M})$, then $g^{\prime}(i)=\mathbf{M}\left(\varphi_{i}\right)$. Let $p_{o}$ be the predecessor function for $O$ (i.e., for all $x$, if $x=2^{y}$, then $p_{o}(x)=y ; p_{o}(x) \uparrow$, otherwise.) Let $h$ be defined as follows. $h\left(i, t_{2}, t_{1}\right)=\max \left(\left\{h^{\prime}\left(x, t_{2}, t_{1}\right) \mid x \leq i\right\}\right)$. Let $g(i)=h(i, \infty, \infty)$. 
We get for all $i, t_{2}, \operatorname{card}\left(\left\{t \mid h\left(i, t_{2}, t\right) \neq h\left(i, t_{2}, t+1\right)\right\}\right) \leq 2(i+1)$; and for all $i, \operatorname{card}(\{t \mid$ $h(i, t, \infty) \neq h(i, t+1, \infty)\}) \leq 2(i+1)$. For all $i, t_{1}$ and $t_{2}$, let

$$
\begin{aligned}
& \mathbf{F}_{1}\left(i, t_{2}, t_{1}\right)= \begin{cases}\frac{2(i+1),}{p_{o}\left(\mathbf{F}_{1}\left(i, t_{2}, t_{1}-1\right)\right),}, & \text { if } t_{1}=0 ; \wedge h\left(i, t_{2}, t_{1}\right) \neq h\left(i, t_{2}, t_{1}-1\right) ; \\
\mathbf{F}_{1}\left(i, t_{2}, t_{1}\right), & \text { otherwise. }\end{cases} \\
& \mathbf{F}_{2}\left(i, t_{2}, t_{1}\right)= \begin{cases}\frac{2(i+1),}{p_{o}\left(\mathbf{F}_{2}\left(i, t_{2}-1, t_{1}\right)\right),} & \text { if } t_{2}=0 ; \\
\mathbf{F}_{2}\left(i, t_{2}-1, t_{1}\right), & \text { otherwise. }\end{cases}
\end{aligned}
$$

Let $w_{1}$ and $w_{2}$ be any notations for $\omega$. Clearly, $h, \mathbf{F}_{1}$ and $\mathbf{F}_{2}$ witness that $g$ is a monotonically non-decreasing $\lim _{w_{2}, w_{1}}^{2}$-computable function. Also, $g$ dominates $g^{\prime}$.

Therefore, $\mathcal{S} \in \mathbf{L i m}_{w_{2}, w_{1}}^{2}$ Mex.

\section{Corollary 48}

For any notations $w_{1}$ and $w_{2}$ for $\omega, \mathbf{M e x} \subset \operatorname{LimMex} \subset \mathbf{L i m}_{w_{2}, w_{1}}^{2} \mathbf{M e x}=\mathbf{E x}$.

The following theorem resolves the remaining relationships between $\mathbf{L i m}_{u_{2}, u_{1}}^{2} \mathbf{M e x}$ classes.

\section{Theorem 49 For all $n \geq 0$ : $\operatorname{LimMex}=\mathbf{L i m}_{\underline{n}, *}^{2} \mathbf{M e x}=\operatorname{Lim}_{*, \underline{n}}^{2} \mathbf{M e x}=\operatorname{Lim}_{\underline{1}, \underline{1}}^{2} \mathbf{M e x}$.}

Proof of Theorem 49. We first prove Lemmas 50, 51 and 52. The theorem then follows easily from these lemmas.

Lemma 50 Every lim-computable function $g$ is a lim $_{1, \underline{1}}^{2}$-computable function.

Proof of Lemma 50. Suppose $g$ is lim-computable as witnessed by computable $h$. We define

$$
h_{1}(x, y)= \begin{cases}h(x, y), & \text { if }(\forall t \geq y)[h(x, t)=h(x, y)] \\ 0, & \text { otherwise }\end{cases}
$$

and

$$
h_{2}(x, y, z)= \begin{cases}h(x, y), & \text { if }(\forall t \mid y \leq t \leq y+z)[h(x, y)=h(x, t)] \\ 0, & \text { otherwise. }\end{cases}
$$

Clearly, $h_{2}$ is a computable function. It is easy to verify the following:

(a) For each $x, y, \operatorname{card}\left(\left\{z \mid h_{2}(x, y, z) \neq h_{2}(x, y, z+1)\right\}\right) \leq 1$;

(b) For each $x, \operatorname{card}\left(\left\{y \mid h_{1}(x, y) \neq h_{1}(x, y+1)\right\}\right) \leq 1$;

(c) For all $x, y, h_{2}(x, y, \infty)=h_{1}(x, y)$;

(d) For all $x, h_{1}(x, \infty)=h(x, \infty)=g(x)$.

Clearly, $g$ is $\lim _{\underline{1}, \underline{1}}^{2}$-computable as witnessed by $h_{2}$.

(LEMMA 50)

Lemma 51 Suppose $n \in N$. Then, for each monotonically non-decreasing lim $_{\underline{n}, *}^{2}$-computable function $g$, there exists a lim-computable function $g^{\prime}$ such that $g^{\prime}$ dominates $g$. 
Proof of Lemma 51. By induction on $n$.

The lemma clearly holds for $n=0$. Suppose by induction that the lemma holds for $n=m$. We will show that the lemma holds for $n=m+1$. Therefore, suppose $g$ is a $\lim _{m+1, *}^{2}$-computable function. Hence, there exist $h_{1}$ and computable $h_{2}$ such that the following three conditions are true.

(a) For all $x, y, h_{1}(x, y)=h_{2}(x, y, \infty)$.

(b) For all $x, g(x)=h_{1}(x, \infty)$.

(c) For all $x, \operatorname{card}\left(\left\{y \mid h_{1}(x, y) \neq h_{1}(x, y+1)\right\}\right) \leq m+1$.

Case 1: For all but finitely many $x, \operatorname{card}\left(\left\{y \mid h_{1}(x, y) \neq h_{1}(x, y+1)\right\}\right) \leq m$.

In this case, it is easy to show that $g(x)$ is $\lim _{\underline{m}, *^{*}}^{2}$-computable. To see this, let $y_{0}$ be such that,

$$
\left(\forall x \mid \operatorname{card}\left(\left\{y \mid h_{1}(x, y) \neq h_{1}(x, y+1)\right\}\right)=m+1\right)\left(\forall y \geq y_{0}\right)\left[h_{1}(x, y)=h_{1}\left(x, y_{0}\right)\right]
$$

Let $h_{2}^{\prime}$ be computable, such that for all $x, y$ and $y, h_{2}^{\prime}(x, y, z)=h_{2}\left(x, y+y_{0}, z\right)$. It is easy to verify that $g$ is $\lim _{m, *}^{2}$-computable as witnessed by $h_{2}^{\prime}$. Hence, by the inductive hypothesis, there is a lim-computable $g^{\prime}$ such that $g^{\prime}$ dominates $g$.

Case 2: For infinitely many $x, \operatorname{card}\left(\left\{y \mid h_{1}(x, y) \neq h_{1}(x, y+1)\right\}\right)=m+1$.

We define a function $H$ as follows.

$$
H(x)=\min \left(\left\{\left\langle z, y_{0}, y_{1}, y_{2}, \ldots, y_{m+1}\right\rangle \mid z \geq x \wedge(\forall j \leq m)\left[y_{j}<y_{j+1} \wedge h_{1}\left(z, y_{j}\right) \neq h_{1}\left(z, y_{j+1}\right)\right]\right\}\right)
$$

$H$ is lim-computable since, for each $x$, the characteristic function of the set, $\left\{\left\langle z, y_{0}, y_{1}, y_{2}, \ldots, y_{m+1}\right\rangle \mid z \geq x \wedge(\forall j \leq m)\left[y_{j}<y_{j+1} \wedge h_{1}\left(z, y_{j}\right) \neq h_{1}\left(z, y_{j+1}\right)\right]\right\}$ is lim-computable.

We next use $H$ to define $g^{\prime}$ satisfying the requirements of the lemma. First define $H_{1}, H_{2}$ as follows: suppose $H(x)=\left\langle z, y_{0}, y_{1}, \ldots, y_{m+1}\right\rangle$; then let $H_{1}(x)=z$ and $H_{2}(x)=y_{m+1}$. Clearly, $H_{1}, H_{2}$ are lim-computable functions. Now it is easy to verify that, if $x \leq x^{\prime}$, then $H_{1}(x) \leq H_{1}\left(x^{\prime}\right)$. Let $g^{\prime}(x)=h_{1}\left(H_{1}(x), H_{2}(x)\right)=g\left(H_{1}(x)\right)$. Since $h_{1}, H_{1}, H_{2}$ are lim-computable functions, it follows that $g^{\prime}$ is also lim-computable and dominates $g$.

(LEMMA 51)

Lemma 52 Every $\lim _{*, \underline{n}}^{2}$-computable function $g$ is $\lim _{\underline{n}, *^{-}}^{2}$ computable.

Proof of Lemma 52. Let $h_{1}, h_{2}$ be such that

(a) for all $x, g(x)=h_{1}(x, \infty)$;

(b) for all $x, y, h_{1}(x, y)=h_{2}(x, y, \infty)$;

(c) for all $x, y, \operatorname{card}\left(\left\{t \mid h_{2}(x, y, t) \neq h_{2}(x, y, t+1)\right\}\right) \leq n$.

For all $i, x, y$, we define

$$
G_{2}^{i}(x, y)=\left\{\begin{array}{cc}
h_{2}(x, y, z), & \text { if } \operatorname{card}\left(\left\{t \mid h_{2}(x, y, t) \neq h_{2}(x, y, t+1)\right\}\right) \geq i \text { and } z=1+ \\
& \min \left(\left\{z^{\prime} \mid \operatorname{card}\left(\left\{t \leq z^{\prime} \mid h_{2}(x, y, t) \neq h_{2}(x, y, t+1)\right\}\right)\right.\right. \\
& \geq i\}) ; \\
\uparrow, & \text { otherwise. }
\end{array}\right.
$$

Intuitively, $G_{2}^{i}$, for each $x, y$ assumes that $h_{2}(x, y, \cdot)$ makes exactly $i$ mind changes. Based on this assumption, it tries to compute $\lim _{t \rightarrow \infty} h_{2}(x, y, t)$.

We note that $\max \left(\left\{i \leq n \mid \operatorname{card}\left(\left\{z \mid G_{2}^{i}(x, z) \downarrow\right\}\right) \geq y\right\}\right)$, is a lim-computable function in $x, y$. Let $X$ be a computable function such that $X(x, y, \infty)=\max \left(\left\{i \leq n \mid \operatorname{card}\left(\left\{z \mid G_{2}^{i}(x, z) \downarrow\right\}\right) \geq y\right\}\right)$. $X(x, y, \infty)$ is monotonically non-increasing in $y$ and is bounded by $n$. Moreover, for all $x$, for all $i>X(x, \infty, \infty), \operatorname{card}\left(\left\{z \mid G_{2}^{i}(x, z) \downarrow\right\}\right)$ is finite. 
We recall from Section 2.1 that $\max (\emptyset)=0$. Let $H(x, y, z)=G_{2}^{X(x, y, z)}(x, w)$, where $w=$ $\max \left(\left\{w^{\prime} \leq z \mid G_{2}^{X(x, y, z)}\left(x, w^{\prime}\right) \downarrow\right.\right.$ in $\leq z$ steps $\left.\}\right)$. Then, for all $x, \operatorname{card}(\{y \mid H(x, y, \infty) \neq H(x, y+$ $1, \infty)\}) \leq n$. Moreover, using the definition of $X, G_{2}^{X(x, \infty, \infty)}$ and $H$, it is easy to verify that $H(x, \infty, \infty)=h_{2}(x, \infty, \infty)=g(x)$. Lemma follows.

(LEMMA 52)

From Lemma 50, we get $\mathbf{L i m M e x} \subseteq \mathbf{L i m}_{1,1}^{2} \mathbf{M e x}$. Clearly, for any lim-computable $g^{\prime}$, there exists a monotonically non-decreasing lim-computable $g^{\prime \prime}$ such that $g^{\prime \prime}$ dominates $g^{\prime}$. Hence, by Lemma 51, we get that for all $n, \mathbf{L i m}_{\underline{n}, *}^{2} \operatorname{Mex} \subseteq \operatorname{LimMex.~Finally,~by~Lemma~} 52$, for all $n$, $\mathbf{L i m}_{*, \underline{n}}^{2} \mathbf{M e x} \subseteq \mathbf{L i m}_{\underline{n}, *}^{2} \mathbf{M e x}$. Thus, the theorem follows from the above three lemmas.

(THEOREM 49)

Thus, if both $u_{1}$ and $u_{2}$ are $\underline{0}, \mathbf{L i m}_{u_{2}, u_{1}}^{2} \mathbf{M e x}=\mathbf{M e x}$. Else, when both of $u_{1}, u_{2}$ are not equal to $\underline{0}, \mathbf{L i m}_{u_{2}, u_{1}}^{2}$ Mex is equal to either LimMex or Ex; otherwise, if $u_{i}$ is the member of $\left\{u_{1}, u_{2}\right\}$ that is not $\underline{0}$, then $\mathbf{L i m}_{u_{2}, u_{1}}^{2} \mathbf{M e x}$ is identical to $\mathbf{L i m}_{u_{i}} \mathbf{M e x}$.

We next briefly consider iterating limits to three levels. A proposition similar to Proposition 46 holds, but we omit stating it here. The following theorem shows that for parsimony factors that use three levels of iterated limits, allowing even a small number of mind changes in each limit essentially retains no parsimony in the final programs learned.

Theorem $53 \mathbf{E x}=\operatorname{Lim}_{\underline{1}, 2,2}^{3} \operatorname{Mex}$.

Proof of TheOrem 53. We first prove Lemma 54 below.

Lemma 54 Suppose $f$ is a computable function from $N^{2} \rightarrow N$. Let $F(i)=\lim _{t \rightarrow \infty} f(i, t)$. Then, there exists a lim li,, $2_{2}^{3}$ function $g$ such that, for all $i, g(i)=\max (\{F(j) \mid j \leq i \wedge F(j) \downarrow\})$.

Proof of Lemma 54. Suppose $f, F$ are given as in the lemma. Below, we define $g(),. h_{1}(.,),. h_{2}(., .,$.$) and h_{3}(., ., .,$.$) (where h_{3}$ is computable), such that each of the following seven clauses is true.

(a) For all $i, g(i)=h_{1}(i, \infty)$;

(b) For all $i, n, h_{1}(i, n)=h_{2}(i, n, \infty)$.

(c) For all $i, n, m, h_{2}(i, n, m)=h_{3}(i, n, m, \infty)$.

(d) For all $i, \operatorname{card}\left(\left\{n \mid h_{1}(i, n) \neq h_{1}(i, n+1)\right\}\right) \leq 1$.

(e) For all $i, n, \operatorname{card}\left(\left\{m \mid h_{2}(i, n, m) \neq h_{2}(i, n, m+1)\right\}\right) \leq 2$.

(f) For all $i, n, m, \operatorname{card}\left(\left\{t \mid h_{3}(i, n, m, t) \neq h_{3}(i, n, m, t+1)\right\}\right) \leq 2$.

(g) $g(i)=\max (\{F(j) \mid j \leq i \wedge F(j) \downarrow\})$.

The lemma then easily follows from the above clauses. We now define $h_{3}$.

Let $X_{3, n, m, t}^{i, n}=\{j \leq i \mid(\forall w \leq m)[f(j, n)=f(j, n+w)]\}$.

Let $Y_{3}^{i, n, m, t}=\{j \leq i \mid(\forall w \leq m+t)[f(j, n)=f(j, n+w)]\}$.

Let $Z_{3}^{i, n, m, t}=\{j \leq i \mid(\exists w \leq t)[f(j, n+m) \neq f(j, n+m+w)]\}$.

Let $P_{3}(i, n, m, t) \equiv\left[\left(X_{3}^{i, n, m, t}=Y_{3}^{i, n, m, t}\right)\right.$ and $\left.\left(X_{3}^{i, n, m, t} \cup Z_{3}^{i, n, m, t}=\{x \mid x \leq i\}\right)\right]$.

$$
h_{3}(i, n, m, t)= \begin{cases}\max \left(\left\{f(j, n) \mid j \in X_{3}^{i, n, m, t}\right\}\right), & \text { if } P_{3}(i, n, m, t) ; \\ 0, & \text { otherwise. }\end{cases}
$$

If $i, n$ and $m$ are fixed, $Y_{3}^{i, n, m, t}$ is monotonically non-increasing and $Z_{3}^{i, n, m, t}$ is monotonically non-decreasing in $t$ (in the set containment sense). $X_{3}^{i, n, m, t}$ is, clearly, fixed once $i, m$ and $n$ are. 
Thus, if $t<t^{\prime}<t^{\prime \prime}$, then $P_{3}(i, n, m, t)$ and $\neg P_{3}\left(i, n, m, t^{\prime}\right)$ implies $\neg P_{3}\left(i, n, m, t^{\prime \prime}\right)$. So (f) is true. Let

$$
\begin{aligned}
& X_{2}^{i, n, m}=X_{3}^{i, n, m, \infty}=X_{3}^{i, n, m, 0}=\{j \leq i \mid(\forall w \leq m)[f(j, n)=f(j, n+w)]\} . \\
& Y_{2}^{i, n, m}=Y_{3}^{i, n, m, \infty}=\{j \leq i \mid(\forall w)[f(j, n)=f(j, n+w)]\} . \\
& Z_{2}^{i, n, m}=Z_{3}^{i, n, m, \infty}=\{j \leq i \mid(\exists w)[f(j, n+m) \neq f(j, n+m+w)]\} . \\
& P_{2}(i, m, n)=P_{3}(i, n, m, \infty) \equiv\left[\left(X_{2}^{i, n, m}=Y_{2}^{i, n, m}\right) \text { and }\left(X_{2}^{i, n, m} \cup Z_{2}^{i, n, m}=\{x \mid x \leq i\}\right)\right] .
\end{aligned}
$$

Let $h_{2}(i, n, m)=h_{3}(i, n, m, \infty)$. Thus (c) holds. It is easy to verify that

$$
h_{2}(i, n, m)= \begin{cases}\max \left(\left\{f(j, n) \mid j \in X_{2}^{i, n, m}\right\}\right), & \text { if } P_{2}(i, n, m) \\ 0, & \text { otherwise }\end{cases}
$$

Now $X_{2}^{i, n, m}$ is a monotonically non-increasing function of $m, Y_{2}^{i, n, m}$ is independent of $m$, and $Z_{2}^{i, n, m}$ is a monotonically non-increasing function of $m$. Thus, if $m<m^{\prime}<m^{\prime \prime}$, then $P_{2}(i, n, m)$ and $\neg P_{2}\left(i, n, m^{\prime}\right)$ implies $\neg P_{2}\left(i, n, m^{\prime \prime}\right)$. It immediately follows that (e) holds. Let

$$
\begin{aligned}
& X_{1}^{i, n}=X_{2}^{i, n, \infty}=\{j \leq i \mid(\forall w)[f(j, n)=f(j, n+w)]\} . \\
& Y_{1}^{i, n}=Y_{2}^{i, n, \infty}=Y_{2}^{i, n, 0}=\{j \leq i \mid(\forall w)[f(j, n)=f(j, n+w)]\}, \\
& Z_{1}^{i, n}=Z_{2}^{i, n, \infty}=\{j \leq i \mid F(j) \uparrow\} . \\
& P_{1}(i, n)=P_{2}(i, n, \infty) \equiv\left[X_{1}^{i, n}=Y_{1}^{i, n} \text { and } X_{1}^{i, n} \cup Z_{1}^{i, n}=\{x \mid x \leq i\}\right]
\end{aligned}
$$

Let $h_{1}(i, n)=h_{2}(i, n, \infty)$. Thus (b) holds.

It is easy to verify that

$$
h_{1}(i, n)= \begin{cases}\max \left(\left\{f(j, n) \mid j \in X_{1}^{i, n}\right\}\right), & \text { if } P_{1}(i, n) \\ 0, & \text { otherwise }\end{cases}
$$

Note that $X_{1}^{i, n}=Y_{1}^{i, n}$ always holds. Thus $P_{1}(i, n)$ is equivalent to $X_{1}^{i, n}=\{j \leq i \mid F(j) \downarrow\}$.

Hence,

$$
h_{1}(i, n)= \begin{cases}\max (\{f(j, n) \mid j \leq i \wedge F(j) \downarrow\}), & \text { if } P_{1}(i, n) \\ 0, & \text { otherwise. }\end{cases}
$$

Note that for all $i, n, n^{\prime}$ such that $n<n^{\prime}$, if $P_{1}(i, n)$ then $P_{1}\left(i, n^{\prime}\right)$. Thus (d) holds. Let $g(i)=\lim _{n \rightarrow \infty} h_{1}(i, n)$. Thus (a) holds. Now, $g(i)=h_{1}(i, \infty)=\max (\{F(j) \mid j \leq i \wedge F(j) \downarrow\})$. Thus (g) holds.

Since, for every machine $\mathbf{M}$ there exists a computable function $f$ such that, $\left(\forall i \mid \varphi_{i} \in\right.$ $\mathbf{E x}(\mathbf{M}))\left[f(i, \infty)=\mathbf{M}\left(\varphi_{i}\right)\right]$ the theorem follows.

There are many mostly uninvestigated questions still open. For $u \in O$, do the $\mathbf{L i m}_{\underline{u}} \mathbf{M e x}$ criteria have "limiting-standardizability" style characterizations similar to those first obtained for Mex in [Fre75]. Generally, except for the cases noted above and their easy consequences, for $u_{1}, \ldots, u_{n} \in(O \cup\{*\})$, how do the learning classes $\mathbf{L i m}_{u_{n}, \ldots, u_{1}}^{n}$ Mex compare to one another?

\subsection{Notation Dependence Results}

As mentioned in Section 1.2, Case and Suraj [CS03] show how to characterize the $\lim _{u}$-computable functions and variants in terms of concepts from [EHK81, Sel84]. One of these characterizations is exploited to prove

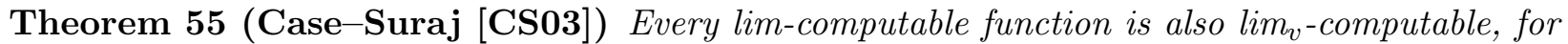
some notation $v$ for $\omega^{2}$. 
Using Theorem 55, we can get the following.

Theorem 56 For all $\mathcal{C}$, if $\mathcal{C} \in \operatorname{LimMex}$ then there exists a notation $v$ for $\omega^{2}$ such that $\mathcal{C} \in$ $\operatorname{Lim}_{v}$ Mex.

Proof. Suppose $\mathcal{C} \in \mathbf{L i m M e x}$ as witnessed by $\mathbf{M}$ and a monotonically non-decreasing limcomputable $g$. By Theorem 55, there exists a notation $v$ for $\omega^{2}$, such that $g$ is $\lim _{v}$-computable. Hence, $\mathcal{C} \in \mathbf{L i m}_{v} \mathbf{M e x}$ as witnessed by the same $\mathbf{M}$ and $g$.

Theorem 56 essentially says that for every class of functions that is LimMex-identifiable, there is some notation $v$ for an ordinal as small as $\omega^{2}$, such that this class of functions is $\mathbf{L i m}_{v} \mathbf{M e x}^{-}$ identifiable. However, we note that by General Hierarchy Theorem (Theorem 32), that one may $\mathbf{L i m}_{v^{\prime}}$ Mex-identify strictly more classes of functions by using a suitable notation $v^{\prime}>_{o} v$.

Also, by Corollary 34, for all $u \in O$, there exist a class of functions $\mathcal{C}$ such that $\mathcal{C} \in(\mathbf{L i m M e x}-$ $\left.\operatorname{Lim}_{u} \mathbf{M e x}\right)$. Hence, by Theorem 56, we have

Corollary $\mathbf{5 7}$ For each $u \in O$, there exists a notation $v$ for $\omega^{2}$, such that $\mathbf{L i m}_{v} \mathbf{M e x} \nsubseteq \mathbf{L i m}_{u} \mathbf{M e x}$.

Using Corollary 57, we get

Corollary 58 For each constructive ordinal $\alpha$ such that $\alpha \geq \omega^{2}$, there exist notations $u, v$ for $\alpha$ such that $\mathbf{L i m}_{u} \mathbf{M e x} \neq \mathbf{L i m}_{v} \mathbf{M e x}$.

Proof. Suppose $\alpha$ is a constructive ordinal $\geq \omega^{2}$ and $u$ is for $\alpha$. By Corollary 57, we get that there exists a notation $u^{\prime}$ for $\omega^{2}$, such that $\mathbf{L i m}_{u^{\prime}} \mathbf{M e x} \nsubseteq \mathbf{L i m}_{u} \mathbf{M e x}$.

Let $v$ be a notation for $\alpha$ such that $u^{\prime} \leq_{o} v \leq_{o}\left(u^{\prime}+_{o} u\right)$; as noted in Section 2.3, such a notation exists, and by Fact 7, it is unique. By Proposition 19, $\mathbf{L i m}_{u^{\prime}} \mathbf{M e x} \subseteq \mathbf{L i m}_{v} \mathbf{M e x}$; therefore, $\operatorname{Lim}_{v} \operatorname{Mex} \nsubseteq \operatorname{Lim}_{u}$ Mex. Hence the corollary follows.

We do not know if there are $u, v \in O$ such that $\operatorname{Lim}_{u} \operatorname{Mex} \subset \operatorname{Lim}_{v} \operatorname{Mex}$ and yet $|u|_{o}>|v|_{o}$.

Also from [CS03], we have

Theorem 59 (Case-Suraj [CS03]) For all $u, v \in O$, such that $u$ and $v$ are for the same ordinal $<\omega^{2}$, every lim $_{u}$-computable function is also lim $_{v}$-computable.

From Theorem 59, we get

Theorem 60 For all $u, v \in O$, such that $u$ and $v$ are for the same ordinal $<\omega^{2}, \mathbf{L i m}_{u} \mathbf{M e x}=$ $\operatorname{Lim}_{v}$ Mex.

It is interesting to ask what happens if we base parsimony restricted criteria on constructive ordinals instead of on notations in $O$ for them. One way to do so is to let, for any constructive ordinal $\alpha$,

$$
\operatorname{Lim}_{\alpha} \operatorname{Mex} \stackrel{\text { def }}{=} \bigcup_{u \text { for } \alpha} \operatorname{Lim}_{u} \text { Mex. }
$$

While we have seen that the criteria $\operatorname{Lim}_{u} \mathbf{M e x}$, for $u \in O$, form subtle and finely graded and ramified infinite hierarchies, there are but three distinct criteria of the form $\mathbf{L i m}_{\alpha} \mathbf{M e x}$, for constructive ordinals $\alpha$, and they form a linear, finite hierarchy thus.

\section{Theorem 61}

(a) For all constructive ordinals $\alpha$ such that $\alpha \geq \omega^{2}, \mathbf{L i m}_{\alpha} \mathbf{M e x}=\mathbf{L i m M e x}$.

(b) $\operatorname{Mex} \subset \operatorname{Lim}_{\omega} \operatorname{Mex} \subset \operatorname{Lim}_{\omega^{2}} \operatorname{Mex}$. 
ProOF.

From Theorem 56, we get $\mathbf{L i m M e x} \subseteq \mathbf{L i m}_{\omega^{2}}$ Mex. From Proposition 19, we get $\mathbf{L i m}_{\omega^{2}} \mathbf{M e x} \subseteq$ LimMex. Hence Part (a) follows.

(b) Follows from Theorems 60, 40 and 23.

We have not fully considered the possible dependencies of the $\lim _{u}$-computable functions or of the classes $\mathbf{L i m}_{u} \mathbf{M e x}$ on the many notation systems alternative to $O([\mathrm{Amb95}])$. Of course, for example, by an obvious embedding, our Strong Hierarchy Theorem (Theorem 40 just above) mutatis mutandis clearly follows for computably related, non maximal ([Rog67]) notation systems based on exponential polynomials in finite ordinals, $\omega$, and a suitably specified collection of $\epsilon$ numbers. Also, we have not considered parsimony restricted criteria based on notations or programs for non-well orderings (with, for example, no computable infinite descending chains) [Ers68b, SSV97, AFS99].

\subsection{Comparison with $O$-Bounded Mind Change Ex-Identification}

We recall that SEG is the set of all finite initial sequences of natural numbers. We next consider, for $u, v \in O$, connections between the $\operatorname{Lim}_{v} \mathbf{M e x}$ criteria and the $\mathbf{E x}_{u}$ criteria that were introduced by Freivalds and Smith [FS93]. To define $\mathbf{E x}_{u}$, we first define $O$-mind change counters, similar to those defined in [JS97, AJS99]: an $O$-mind change counter is a computable mapping, F, from SEG into $O$, such that for all $n$ and $f, \mathbf{F}(f[n]) \geq_{o} \mathbf{F}(f[n+1])$. Now, for each $u \in O$, a machine $\mathbf{M} \mathbf{E x}_{u}$-identifies $f$ if and only if $\mathbf{M}$ (i) Ex-identifies $f$, and (ii) there exists an $O$-mind change counter $\mathbf{F}$ such that for every $n,[? \neq \mathbf{M}(f[n]) \neq \mathbf{M}(f[n+1])] \Rightarrow \mathbf{F}(f[n+1])<_{o} \mathbf{F}(f[n]) \leq_{o} u$. As can be seen, the $\mathbf{E x}_{u}$ criteria impose restrictions on the mind changes of the learning machines themselves.

Besides [FS93], other works that study various aspects of imposing similar restrictions on the mind changes of learning machines include those of Apsitis [Aps94], Ambainis [Amb95], Jain and Sharma [JS97, JS99, JS01], Sharma, Stephan and Ventsov [SSV97], Ambainis, Freivalds and Smith [AFS99] and, Ambainis, Jain, and Sharma [AJS99].

Theorem 62 Mex $\nsubseteq \bigcup_{u \in O} \mathbf{E x}_{u}$.

Proof of Theorem 62. Let $\mathcal{C}=\left\{f \mid\left(\forall^{\infty} x\right)[f(x)=0] \wedge 2 * \operatorname{MinProg}(f) \geq \max (\{x \mid f(x) \neq\right.$ $0\} \cup\{f(x) \mid x \in N\})\}$.

Now, for any finite set $S \subseteq N \times N$, where, $S$ is single-valued, i.e., $S$ is the graph of a finite partial function, let $\operatorname{pzext}(S)$ be a standard program for the zero-extension of $S$, i.e.,

$$
\varphi_{\operatorname{pzext}(S)}(x)= \begin{cases}y, & \text { if }(x, y) \in S \\ 0, & \text { if there is no } y \text { with }(x, y) \in S .\end{cases}
$$

Let $g(z)=\max (\operatorname{pzext}(S) \mid S \subseteq\{0, \cdots, 2 z\} \times\{0, \cdots, 2 z\}$ and $S$ is single-valued).

Let $\mathbf{M}(f[n])=\operatorname{pzext}(\{(x, f(x)) \mid x<n \wedge f(x) \neq 0\})$.

Clearly, for any $\varphi_{e} \in \mathcal{C}, g(e)$ bounds $\operatorname{pzext}(S)$, for $S=\left\{\left(x, \varphi_{e}(x)\right) \mid \varphi_{e}(x) \neq 0\right\}$. Hence $\mathbf{M}$ $g$-Mex-identifies $\mathcal{C}$.

We next show that, for all $u \in O, \mathcal{C} \notin \mathbf{E x}_{u}$. 
Note first that $\mathcal{C}$ is dense, i.e., for any element $f[n] \in$ SEG, there exists an extension $g$ of $f[n]$ such that $g \in \mathcal{C}$. To see this, let $z=1+\max (\{n\} \cup\{f(x) \mid x<n\})$. For each $y$, let

$$
g_{y}(x)= \begin{cases}f(x), & \text { if } x<n \\ y, & \text { if } x=n \\ 0, & \text { otherwise }\end{cases}
$$

Now, there exists a $y \in\{x \mid z \leq x \leq 2 z\}$, such that $\operatorname{MinProg}\left(g_{y}\right) \geq z$. Thus $g_{y} \in \mathcal{C}$.

Now suppose by way of contradiction that, for some $u \in O, \mathcal{C} \in \mathbf{E x}_{u}$ as witnessed by some machine $\mathbf{M}^{\prime}$ and $O$-mind change counter $\mathbf{F}$. Since for every $n,\left[? \neq \mathbf{M}^{\prime}(f[n]) \neq \mathbf{M}^{\prime}(f[n+1])\right] \Rightarrow$ $\mathbf{F}(f[n+1])<_{o} \mathbf{F}(f[n]) \leq_{o} u$, and there are no infinite descending chains of notations in $O$, we get that $\mathbf{M}^{\prime}$ makes only finitely many mind changes on any $f$. Therefore, there is a $\sigma$ such that $\mathbf{M}^{\prime}$ does not make a mind change on any $\tau$ that is an extension of $\sigma$. Clearly, since $\mathcal{C}$ is dense, there are infinitely many $f^{\prime}$ 's in $\mathcal{C}$ that are each extensions of $\sigma$; yet, $\mathbf{M}^{\prime} \mathbf{E x}_{u}$-identifies at most one of them. Therefore, $\mathbf{M}^{\prime}$ does not $\mathbf{E} \mathbf{x}_{u}$-identify $\mathcal{C}$.

Hence, the theorem follows.

(THEOREM 62)

Theorem 63 For all $u \in O$, for all $w$ for $\left.\left.\omega, \mathbf{E x}_{u} \subset \operatorname{Lim}_{\{(u+o 2}\right)^{w}\right\}_{o}$ Mex.

ProOF.

We show that for all $u$, for all $w$ for $\left.\left.\omega, \mathbf{E x}_{u} \subseteq \operatorname{Lim}_{\{(u+o 2}\right)^{w}\right\}_{o}$ Mex. Then, the theorem follows using Theorem 62 .

Suppose $\mathcal{C} \in \mathbf{E x}_{u}$ as witnessed by machine $\mathbf{M}$ and $O$-mind change counter $\mathbf{F}^{\prime}$. We first show how to construct a (not necessarily monotonically non-decreasing) $\lim _{u+{ }_{o} \underline{1}}$-computable function $g$, such that, $(\forall f \in \mathcal{C})[g(\operatorname{MinProg}(f) \geq \mathbf{M}(f)]$. In this interest, we present a simple modification of the construction from the proof of Lemma 29 to get $h$ and $\mathbf{F}$ that witness that $g$ is $\lim _{u+{ }_{o} \underline{1}}$-computable.

Let $h$ and $\mathbf{F}$ be defined as follows. For all $i$, let $h(i, 0)=0, \mathbf{F}(i, 0)=u+_{o} \underline{1}$. For all $i, t$, we define $h(i, t+1), \mathbf{F}(i, t+1)$ as follows.

begin computation of $h(i, t+1), \mathbf{F}(i, t+1)$.

if $\Phi_{i}(0)>t$

then

Let $h(i, t+1)=h(i, t)$ and $\mathbf{F}(i, t+1)=\mathbf{F}(i, t)$;

else

Let $x$ be the largest value $\leq t$, such that $\left(\forall x^{\prime} \leq x\right)\left[\Phi_{i}\left(x^{\prime}\right) \leq t\right]$.

Let $h(i, t+1)=\mathbf{M}\left(\varphi_{i}[x+1]\right)$ and $\mathbf{F}(i, t+1)=\mathbf{F}^{\prime}\left(\varphi_{i}[x+1]\right)$.

endif

end computation of $h(i, t+1), \mathbf{F}(i, t+1)$.

Let $g(i)=h(i, \infty)$. Clearly, for all $i$ such that $\varphi_{i} \in \mathcal{C}$, we get $h(i, \infty)=\mathbf{M}(f)$; in particular, for all $f \in \mathcal{C}, h(\operatorname{MinProg}(f), \infty)=\mathbf{M}(f)$. Clearly, also, $h$ and $\mathbf{F}$ witness that $g$ is $\lim _{u+o 1^{-}}$ computable. Suppose $w$ is a notation for $\omega$. Then, by Lemma 25, there exists a monotonically non-decreasing $\lim _{\left\{(u+o \underline{2})^{w}\right\}_{o}}$-computable function $g^{\prime}$ which dominates $g$. It is easy to verify that $\mathcal{C} \in \operatorname{Lim}_{\left\{\left(u+{ }_{o} \underline{2}\right)^{w}\right\}_{o}}$ Mex as witnessed by $\mathbf{M}$ and $g^{\prime}$. Hence, $\mathbf{E x}_{u} \subset \operatorname{Lim}_{\left\{\left(u+{ }_{o} \underline{2}\right)^{w}\right\}_{o}} \mathbf{M e x}$. 
Theorem 64 Suppose $u, v, w \in O$ such that $u<_{o}\left\{w^{v}\right\}_{o}$ and $w$ is for $\omega$. Then, $\mathbf{E x}_{u} \subset \operatorname{Lim}_{\left\{w^{v}\right\}_{o}} \mathbf{M e x}$.

ProOF.

We show that for all $u, v, w \in O$ such that $u<_{o}\left\{w^{v}\right\}_{o}$ and $w$ is for $\omega, \mathbf{E x}_{u} \subseteq \mathbf{L i m}_{\left\{w^{v}\right\}_{o}} \mathbf{M e x}$. Then, the theorem follows using Theorem 62 .

If $v=\underline{0}$, then $\left\{w^{v}\right\}_{o}=\underline{1}$. Therefore, $\mathbf{L i m}_{\left\{w^{v}\right\}_{o}} \mathbf{M e x}=\mathbf{L i m}_{\underline{1}} \mathbf{M e x}$. From Corollary 23, $\mathbf{L i m}_{\underline{1}} \mathbf{M e x}=\operatorname{Mex}$. Also, $\mathbf{E x}_{\underline{0}} \subseteq \operatorname{Mex}[$ Che82, Theorem 6.1, page 80]. Therefore, the $v=\underline{0}$ case follows.

The $v>_{o} \underline{0}$ case is similar to the proof of Theorem 63, except that it makes use of Lemma 37 instead of Lemma 25 and the fact that $u<_{o}\left\{w^{v}\right\}_{o} \Rightarrow u+_{o} \underline{1}<_{o}\left\{w^{v}\right\}_{o}$. We omit the details.

As a corollary to the theorem just above, we get

Corollary 65 Suppose $v, w \in O$ such that $w$ is for $w$. Then, $\mathbf{E x}_{\left\{w^{v}\right\}_{o}} \subset \mathbf{L i m}_{\left\{w^{v}\right\}_{o} \times_{o} w} \mathbf{M e x}$.

For $n \in N^{+}$, Theorem 63 implies for all notations $w$ for $\omega, \mathbf{E x}_{\underline{n}} \subset \mathbf{L i m}_{\left\{\left(\underline{n}+{ }_{o} \underline{2}\right)^{w}\right\}_{o}} \mathbf{M e x}$; the latter, by Theorem 60 and the fact that $\left|\left\{\left(\underline{n}+_{o} \underline{2}\right)^{w}\right\}_{o}\right|_{o}=\omega$, is $=\mathbf{L i m}_{\omega} \mathbf{M e x}$. Chen [Che82, Corollary 6.2, pages 80-81] shows that, in fact, $\mathbf{E x}_{\underline{n}} \subset \mathbf{M e x}$; hence $\mathbf{E x}_{\underline{n}} \subset \mathbf{L i m}_{0} \mathbf{M e x}$. However,

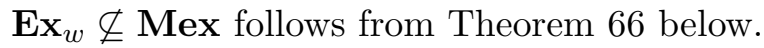

Theorem 66 For all $u, w \in O$ such that $w$ is for $\omega, \mathbf{E x}_{w \times_{o}\left(u+_{o} \underline{1}\right)}-\mathbf{L i m}_{u} \mathbf{M e x} \neq \emptyset$.

ProOF.

Let $u, w \in O$ be such that $w$ is for $\omega$. We use $\mathcal{C}_{u}$ from Definition 28 to show that $\mathcal{C}_{u} \in$ $\mathbf{E x}_{w \times_{o}\left(u+{ }_{o} \underline{1}\right)}-\mathbf{L i m}_{u} \mathbf{M e x}$. The negative part of this follows from Lemma 31 .

The positive part follows, in part, from a careful analysis of a construction from [FW79] as presented in [JORS99]. So that we do not need to present this construction again, in the remainder of this proof we proceed informally to show that $\mathcal{C}_{u} \in \mathbf{E x}_{w \times_{o}(u+o \underline{1})}$.

For $f \in \mathcal{C}_{u}$, from Parts (a) through (e) of Definition 28, we see that $\lim _{x \rightarrow \infty} \pi_{2}^{4}(f(x)) \downarrow \geq \mathrm{a}$ program for $f$, and that this convergence involves $\leq_{o} u$ mind changes. Hence, informally, the number of different values of $\pi_{2}^{4}(f(x))$ on the way to convergence is $\leq_{o}\left(u+_{o} \underline{1}\right)$.

Proposition 10.7 of [JORS99, Page 227] generalizes a result from [FW79], and careful examination of the proof of this Proposition (for the special case from [FW79]) provides the existence of a machine we'll call $\mathbf{M}_{F W}$ with the following useful property. If $\mathbf{M}_{F W}$ receives as input both an upper bound on a program for a function $f$ and successively longer initial segments of $f$, then it converges to a program for $f$. Furthermore, in the process, $\mathbf{M}_{F W}$ makes $\leq_{o} w$ mind changes.

We present informally, then, a machine $\mathbf{M}$ which witnesses that $\mathcal{C}_{u} \in \mathbf{E x}_{w \times_{o}\left(u+{ }_{o}\right)}$. For any $x \in N$ and any function $f, \mathbf{M}$, on $f[x+1]$, employs $\pi_{2}^{4}(f(x))$ as a candidate upper bound on a program for $f$ to feed to $\mathbf{M}_{F W}$ together with $f[x+1]$; then $\mathbf{M}$ outputs $\mathbf{M}_{F W}$ 's resultant output. Each time $\pi_{2}^{4}(f(x))$ makes a mind change, the $\mathbf{M}_{F W}$ component of $\mathbf{M}$ starts over. From above, $\mathbf{M}$ need look at $\leq_{o}\left(u+{ }_{o} \underline{1}\right)$ different values of $\pi_{2}^{4}(f(x))$, and, for each of these, the $\mathbf{M}_{F W}$ component of $\mathbf{M}$ can be restricted to make $\leq_{o} w$ mind changes. Hence, $\mathbf{M}$ makes $\leq_{o}\left(w \times_{o}\left(u+{ }_{o} \underline{1}\right)\right)$ mind changes. To see this, think of $\left|w \times_{o}(u+o \underline{1})\right|_{o}$ informally as $\left(|u|_{o}+1\right)$ copies of $\omega$ laid out end to end; and the counting down for $\mathbf{M}$ as starting at the right end of the rightmost copy of $\omega$, jumping to right end of the next copy of $\omega$ to the left whenever $\pi_{2}^{4}(f(x))$ makes a mind change, and counting down inside its currently employed copy of $\omega$ when operating $\mathbf{M}_{F W}$ between $\pi_{2}^{4}(f(x))$ 's mind changes. 
The notation $\left(w \times_{o}\left(u+_{o} \underline{1}\right)\right)$ makes it possible to find ones way algorithmically from right to left across the corresponding ordinal conceptualized as $\left(|u|_{o}+1\right)$ copies of $\omega$ laid out end to end.

Since for all $u \in O, \operatorname{Mex} \subseteq \mathbf{L i m}_{u}$ Mex, using Theorem 62, we get the following corollary to Theorem 66 above.

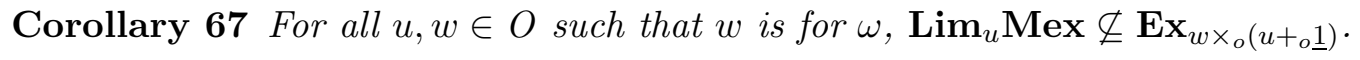

We also note that the proof of Theorem 66 may be easily adapted to show that for all $u, v \in O$, such that $|v|_{o} \geq \omega$, we get $\mathbf{E x}_{v \times_{o}\left(u+_{o} \underline{1}\right)}-\operatorname{Lim}_{u} \mathbf{M e x} \neq \emptyset$.

It is open whether the comparisons of this section can be substantially improved.

\section{References}

[Add65] J. W. Addison. The method of alternating chains. In J. W. Addison, Leon Henkin, and Alfred Tarski, editors, Theory of Models (Proc. 1963 International Symposium, Berkeley, Calif.), pages 1-16, Amsterdam, 1965. North-Holland.

[AFS99] A. Ambainis, R. Freivalds, and C. H. Smith. Inductive inference with procrastination: Back to definitions. Fundamenta Informaticae, 40:1-16, 1999.

[AJS99] A. Ambainis, S. Jain, and A. Sharma. Ordinal mind change complexity of language identification. Theoretical Computer Science, 220(2):323-343, 1999.

[Amb95] A. Ambainis. The power of procrastination in inductive inference: How it depends on used ordinal notations. Lecture Notes in Computer Science, 904:99-111, 1995.

[Ang80] D. Angluin. Finding patterns common to a set of strings. Journal of Computer and System Sciences, 21:46-62, 1980.

[Aps94] Kalvis Apsitis. Derived sets and inductive inference. In Setsuo Arikawa and Klaus P. Jantke, editors, Algorithmic Learning Theory, Proc. 4th International Workshop on Analogical and Inductive Inference (AII'94) and the 5th International Workshop on Algorithmic Learning Theory (ALT'94), October 10-15, 1994, Reinhardsbrunn Castle, Germany, volume 872 of LNAI, pages 26-39. Springer-Verlag, 1994.

[ASY92] S. Arikawa, T. Shinohara, and A. Yamamoto. Learning elementary formal systems. Theoretical Computer Science, 95:97-113, 1992.

[BB75] L. Blum and M. Blum. Toward a mathematical theory of inductive inference. Information and Control, 28:125-155, 1975.

[Beh97] Libor Behounek. Ordinal calculator, 1997. Web document at: http://www.ff.cuni.cz/ behounek/ordinalc.htm.

[Blu67] M. Blum. A machine independent theory of the complexity of recursive functions. Journal of the ACM, 14:322-336, 1967.

[BM95] I. Bratko and S. Muggleton. Applications of inductive logic programming. Communications of the ACM, 38(11):65-70, 1995. 
[BUV96] A. Brazma, E. Ukkonen, and J. Vilo. Discovering unbounded unions of regular pattern languages from positive examples. In Proceedings of the Seventh International Symposium on Algorithms and Computation (ISAAC'96), volume 1178 of Lecture Notes in Computer Science, pages 95-104, 1996. Osaka, Japan.

[Cas74] J. Case. Periodicity in generations of automata. Mathematical Systems Theory, 8:15-32, 1974.

[Cas94] J. Case. Infinitary self-reference in learning theory. Journal of Experimental and Theoretical Artificial Intelligence, 6:3-16, 1994.

[Che82] K. Chen. Tradeoffs in inductive inference of nearly minimal sized programs. Information and Control, 52:68-86, 1982.

$\left[\mathrm{CJK}^{+} 01\right]$ J. Case, S. Jain, S. Kaufmann, A. Sharma, and F. Stephan. Predictive learning models for concept drift. Theoretical Computer Science, 268:323-349, 2001. Special Issue for ALT'98.

[CJLZ99] J. Case, S. Jain, S. Lange, and T. Zeugmann. Incremental concept learning for bounded data mining. Information and Computation, 152:74-110, 1999.

[CJS95] J. Case, S. Jain, and M. Suraj. Not-so-nearly-minimal-size program inference. In Klaus P. Jantke and Steffen Lange, editors, Algorithmic Learning for Knowledge-Based Systems, volume 961 of Lecture Notes in Artificial Intelligence, pages 77-96. Springer-Verlag, 1995.

[CJS96] J. Case, S. Jain, and A. Sharma. Machine induction without revolutionary changes in hypothesis size. Information and Computation, 128:73-86, August 1996.

[CS83] J. Case and C. Smith. Comparison of identification criteria for machine inductive inference. Theoretical Computer Science, 25:193-220, 1983.

[CS03] J. Case and M. Suraj. Characterizing Ershov hierarchies by algorithmic $O$-count down, 2003. Working paper.

[EHK81] R. L. Epstein, R. Haas, and R. L. Kramer. Hierarchies of sets and degrees below $\mathbf{0}^{\prime}$. In Logic Year 1979-80, volume 859 of Lecture Notes in Mathematics, pages 32-48, Heidelberg, 1981. Springer-Verlag.

[Ers68a] Yu. L. Ershov. A hierarchy of sets, I. Algebra i Logika, 7(1):47-74, 1968. In Russian (English translation in Algebra and Logic, 7:25-43, 1968).

[Ers68b] Yu. L. Ershov. A hierarchy of sets, II. Algebra $i$ Logika, 7(4):15-47, 1968. In Russian (English translation in Algebra and Logic, 7:212-232, 1968).

[Ers70] Yu. L. Ershov. A hierarchy of sets, III. Algebra i Logika, 9(1):34-51, 1970. In Russian (English translation in Algebra and Logic, 9:20-31, 1970).

[Fre75] R. Freivalds. Minimal Gödel numbers and their identification in the limit. Lecture Notes in Computer Science, 32:219-225, 1975. 
[Fre90] R. Freivalds. Inductive inference of minimal programs. In M. Fulk and J. Case, editors, Proceedings of the Third Annual Workshop on Computational Learning Theory, pages 3-20. Morgan Kaufmann Publishers, Inc., August 1990.

[FS93] R. Freivalds and C. Smith. On the role of procrastination in machine learning. Information and Computation, 107(2):237-271, 1993.

[Ful85] M. Fulk. A Study of Inductive Inference Machines. PhD thesis, SUNY at Buffalo, 1985.

[FW79] R. Freivalds and R. Wiehagen. Inductive inference with additional information. Electronische Informationverarbeitung und Kybernetik, 15:179-195, 1979.

[GD01] A. Gale and R. Downey. On genericity and Ershov's hierarchy. Mathematical Logic Quarterly, 47(2):161-182, 2001.

[Gol67] E. Gold. Language identification in the limit. Information and Control, 10:447-474, 1967.

[GS95] W. I. Gasarch and C. H. Smith. Recursion theoretic models of learning: some results and intuitions. Annals of Mathematics and Artificial Intelligence, 15(2):151-166, 1995.

[Joc82] C. G. Jockusch, Jr. Review of "Hierarchies of Sets and Degrees Below 0"” by Richard L. Epstein and Richard Haas and Richard L. Kramer. Mathematical Reviews, 1982. MR 82k:03073.

[JORS99] S. Jain, D. Osherson, J. Royer, and A. Sharma. Systems that Learn: An Introduction to Learning Theory. MIT Press, Cambridge, Mass., second edition, 1999.

[JS97] S. Jain and A. Sharma. Elementary formal systems, intrinsic complexity, and procrastination. Information and Computation, 132:65-84, 1997.

[JS99] S. Jain and A. Sharma. Mind change complexity of learning logic programs. In P. Fischer and H.U. Simon, editors, Fourth European Conference on Computational Learning Theory, volume 1572 of Lecture Notes in Artificial Intelligence, pages 198-213. SpringerVerlag, Berlin, 1999.

[JS01] S. Jain and A. Sharma. On a generalized notion of mistake bounds. Information and Computation, 166:156-166, 2001.

[Kin74] E. Kinber. On the synthesis in the limit of almost minimal Gödel numbers. Theory Of Algorithms and Programs, LSU, Riga, 1:221-223, 1974.

[Kle38] S. C. Kleene. On notation for ordinal numbers. Jounal of Symbolic Logic, 3:150-155, 1938.

[Kle44] S. C. Kleene. On the forms of predicates in the theory of constructive ordinals. American Journal of Mathematics, 66:41-58, 1944.

[Kle55] S. C. Kleene. On the forms of predicates in the theory of constructive ordinals (second paper). American Journal of Mathematics, 77:405-428, 1955. 
[KM67] K. Kuratowski and A. Mostowski. Set Theory. North-Holland, 1967.

[KMU95] P. Kilpeläinen, H. Mannila, and E. Ukkonen. MDL learning of unions of simple pattern languages from positive examples. In Paul Vitányi, editor, Second European Conference on Computational Learning Theory, volume 904 of Lecture Notes in Artificial Intelligence, pages 252-260. Springer-Verlag, 1995.

[LD94] N. Lavrač and S. Džeroski. Inductive Logic Programming: Techniques and Applications. Ellis Horwood, 1994.

[Mit97] T. Mitchell. Machine Learning. McGraw Hill, 1997.

[MR94] S. Muggleton and L. De Raedt. Inductive logic programming: Theory and methods. Journal of Logic Programming, 19/20:669-679, 1994.

[MY78] M. Machtey and P. Young. An Introduction to the General Theory of Algorithms. North Holland, New York, 1978.

[Nix83] R. Nix. Editing by examples. Technical Report 280, Department of Computer Science, Yale University, New Haven, CT, USA, 1983.

[Odi99] P. Odifreddi. Classical Recursion Theory, volume II. Elsivier, Amsterdam, 1999.

[Put63] H. Putnam. Probability and confirmation. Voice of America, Forum on Philosophy of Science, 10, 1963.

[Put65] H. Putnam. Trial and error predicates and the solution to a problem of Mostowski. Journal of Symbolic Logic, 30:49-57, 1965.

[Rog58] H. Rogers. Gödel numberings of partial recursive functions. Journal of Symbolic Logic, 23:331-341, 1958.

[Rog67] H. Rogers. Theory of Recursive Functions and Effective Computability. McGraw Hill, New York, 1967. Reprinted, MIT Press, 1987.

[Roy87] J. Royer. A Connotational Theory of Program Structure, volume 273 of Lecture Notes in Computer Science. Springer-Verlag, Berlin, 1987.

[SA95] T. Shinohara and A. Arikawa. Pattern inference. In Klaus P. Jantke and Steffen Lange, editors, Algorithmic Learning for Knowledge-Based Systems, volume 961 of Lecture Notes in Artificial Intelligence, pages 259-291. Springer-Verlag, 1995.

[Sac90] G. Sacks. Higher Recursion Theory. Springer-Verlag, 1990.

[Sal94a] A. Salomaa. Patterns (The Formal Language Theory Column). EATCS Bulletin, 54:46$62,1994$.

[Sal94b] A. Salomaa. Return to patterns (The Formal Language Theory Column). EATCS Bulletin, 55:144-157, 1994.

[Sch98] M. Schaefer. A guided tour of minimal indices and shortest descriptions. Archives for Mathematical Logic, 18:521-548, 1998. 
[Sel84] V. L. Selivanov. On a hierarchy of limiting computations. Sibirskii Mathematicheskii Zhurnal, 25(5):146-156, 1984. In Russian (English translation in Siberian Mathematical Journal, 25:798-806, 1984).

[Sha71] N. Shapiro. Review of "Limiting recursion" by E.M. Gold and "Trial and error predicates and the solution to a problem of Mostowski" by H. Putnam. Journal of Symbolic Logic, 36:342, 1971.

[Shi83] T. Shinohara. Inferring unions of two pattern languages. Bulletin of Informatics and Cybernetics, 20:83-88., 1983.

[Sie65] W. Sierpinski. Cardinal and ordinal numbers. PWN -Polish Scientific Publishers, 1965. Second revised edition.

[Smu61] R. Smullyan. Theory of Formal Systems. Annals of Mathematics Studies, No. 47. Princeton University Press, 1961.

[Soa96] Robert I. Soare. Computability and recursion. Bulletin of Symbolic Logic, 2(3):284-321, 1996.

[SSS $\left.{ }^{+} 94\right]$ S. Shimozono, A. Shinohara, T. Shinohara, S. Miyano, S. Kuhara, and S. Arikawa. Knowledge acquisition from amino acid sequences by machine learning system BONSAI. Trans. Information Processing Society of Japan, 35:2009-2018, 1994.

[SSV97] A. Sharma, F. Stephan, and Y. Ventsov. Generalized notions of mind change complexity. In Proceedings of the Tenth Annual Conference on Computational Learning Theory, pages 96-108. ACM Press, 1997.

[Wri89] K. Wright. Identification of unions of languages drawn from an identifiable class. In R. Rivest, D. Haussler, and M. Warmuth, editors, Proceedings of the Second Annual Workshop on Computational Learning Theory, Santa Cruz, California, pages 328-333. Morgan Kaufmann Publishers, Inc., 1989. 